\title{
BOND PERCOLATION IN FRUSTRATED SYSTEMS
}

\author{
By E. De Santis AND A. Gandolfi \\ Università di Roma La Sopienza and Tor Vergate
}

\begin{abstract}
We study occurrence and properties of percolation of occupied bonds in systems with random interactions and, hence, frustration. We develop a general argument, somewhat like Peierls' argument, by which we show that in $\mathbb{Z}^{d}, d \geq 2$, percolation occurs for all possible interactions (provided they are bounded away from zero) if the parameter $p \in(0,1)$, regulating the density of occupied bonds, is high enough. If the interactions are i.i.d. random variables then we determine bounds on the values of $p$ for which percolation occurs for all, almost all but not all, almost none but some, or none of the interactions. Motivations of this work come from the rigorous analysis of phase transitions in frustrated statistical mechanics systems.
\end{abstract}

1. Introduction. In this paper we study occurrence and properties of percolation of occupied bonds in models, defined on regular lattices (mostly $\mathbb{Z}^{d}$ for simplicity), in which percolation is made more difficult by frustration, which is the presence of particular circuits which cannot be fully occupied. This is determined by restrictions given in terms of a preassigned selection $J$ of interactions, that is, real values (both positive and negative in the interesting cases), one for each bond; the circuits for which the product of the interactions is negative are called frustrated and cannot be fully occupied. We study probability measures on bond occupation variables satisfying this constraint. Two closely related classes of such probability measures, both dependent on one parameter, $p \in[0,1]$, are introduced: a simpler one, which is just a Bernoulli measure conditioned to avoid full occupation of frustrated circuits, and a more interesting one, which is the FK random cluster model (see Section 2). Percolation, that is, the existence, with positive probability with respect to one of the above-mentioned measures, of an infinite connected chain of occupied bonds is then hampered by the need to avoid full occupation of frustrated circuits. Nonetheless, percolation occurs for $p$ sufficiently close to one, for example, $p>8 / 9$ in the conditionally unfrustrated Bernoulli measure and $p>16 / 17$ in the FK random cluster model, for any $J \in\{-1,1\}$ (see Theorems 2.2 and 2.8 below) and in any dimension $d \geq 2$. Occurrence of percolation in such models was not at all clear in dimension 2 even for a set of interactions $J$ having probability 1 according to the Bernoulli distribution (see [13] for the two-dimensional FK random cluster model).

The formulation of the models above is motivated by the role played by the FK random cluster model as a representation of spin systems, in particular of frustrated spin systems like spin glasses. In the ferromagnetic Ising model at zero external field and inverse temperature $\beta$, spontaneous magnetization,

Received May 1997; revised April 1999.

AMS 1991 subject classifications. Primary 60K35; secondary 82A25, 82A57, 82A68.

Key words and phrases. Percolation, frustration, Peierls' argument, spin glasses. 
and so phase transition, occurs if and only if percolation of occupied bonds occurs in the related FK random cluster model with parameter $p=1-e^{-2 \beta}$ (see [10]), but in the spin glass models [8] this correspondence is not as direct (see [23]). In the Edwards-Anderson (EA) spin glass model (see [8]) on the vertices of $\mathbb{Z}^{d}$, for instance, interactions are randomly and independently chosen with some distribution $\mathscr{P}$ symmetric around the origin, and the question is whether phase transition occurs for $\mathscr{P}$-almost all $J$. It is expected (but not proved) that for low enough temperature, that is, high enough $\beta$, phase transition occurs in dimension $d \geq 3$ and it is an open question what to expect in dimension $d=2$. It was proved in [13] that percolation of occupied bonds occurs in the FK random cluster model in $d \geq 3$ for $\mathscr{P}$-almost all $J$ (for at least some simple distribution $\mathscr{P}$ ), and this seemed an indication that the picture outlined above could hold. Here, however, we prove that a similar transition to percolation occurs in dimension $d=2$ also, so that percolation gives no clear indication on whether a phase transition could take place in the EA model (on the relation between percolation and phase transition for spin glasses see also [12]). On the other hand, it is possible to modify the proof of our main result to show that a ferromagnetic phase transition does take place at low density of antiferromagnetic interactions, obtaining a different proof of some of the results in [6]. These being the motivations, we now restrict our attention to percolation of occupied bonds.

Our method in Section 2 shows that for $p$ large enough percolation of occupied bonds occurs, even subject to avoiding full occupation of frustrated circuits, for all $J$. We think that this method is, by itself, quite general, somewhat like Peierls' argument, and we think that it might be applicable to discuss percolation of a variety of possibly frustrated systems; in particular, since the argument applies to all interactions $J$, it can be used in models where a specific nonrandom form of the $J$ is assumed. To illustrate the method, we apply it to the simplest model in Theorem 2.2 and leave further refinements to later parts of the paper.

Given a Bernoulli distribution $\mathscr{P}$ on the interactions $J$, a new phenomenon is described in Section 3: for some values of $p$, percolation might occur for $\mathscr{P}$-almost none but some, or for $\mathscr{P}$-almost all but not all interactions $J$ (these two possibilities, together with the presence or absence of percolation for all $J$ at some other values of $p$, are shown to occur in Theorem 4.2 for the triangular lattice $T$ ). Theorem 3.2 shows that also in the more interesting twodimensional FK random cluster model (with two-valued $J$ ) there are regions of $p$ for which percolation occurs for $\mathscr{P}$-almost none but some J. Questions about statements for all versus almost all realizations, as function of some parameter, have been dealt with in a variety of contexts concerning spatial random systems: random coverings [27], Brownian motion [20], uniqueness of percolation cluster [3] and occurrence of percolation under some evolution [16]. In analogy with some of the results of these papers, we find nontrivial regions which could be called critical, that is, in which not all interactions $J$ behave the same.

Other results concerning presence or absence of percolation for not necessarily two-valued distributions are discussed in Section 4. 
There remain various other issues which are not discussed in the paper. For example, the determination of the influence of the type of transition that we have found on the thermodynamical properties of spin glass systems, in terms for instance of the change in magnetization or correlation, or, more specifically, about percolation of unfrustrated bonds, the determination (of at least the existence) of the critical value for percolation for all interactions $J$ and of the $\mathscr{P}$-dependent critical values for percolation for $\mathscr{P}$-almost all $J$. For further discussion on the regions in which percolation occurs, see [12].

2. Percolation for all interactions. The results presented in this section are quite general and can be applied to a variety of models; some of these applications are exploited in the following sections. Here we present the main ideas in their simplest form, referring to a very simple model which basically just captures the idea of (randomized) frustration.

First we need some geometrical definitions, which are given directly in $\mathbb{Z}^{d}$ for simplicity. Let $|\cdot|$ indicate the Euclidean distance (we use the same symbol later to indicate cardinality of sets, but no confusion should arise), and consider $\mathbb{Z}^{d} \subset \mathbb{R}^{d}$ as a graph with set of vertices $V\left(\mathbb{Z}^{d}\right)=\mathbb{Z}^{d}$ and set of nearest neighbour bonds $B\left(\mathbb{Z}^{d}\right)=\left\{\{i, j\}: i, j \in \mathbb{Z}^{d},|i-j|=1\right\}$. For a bond $b=\{i, j\}$, the vertices $i$ and $j$ are called end points; for a set $E$ of bonds, $\operatorname{Vert}(E) \subseteq \mathbb{Z}^{d}$ is the set of vertices which are end points of at least one bond of $E$. Also, the origin of $\mathbb{Z}^{d}$ is denoted by $O$. We define a path to be an ordered sequence $\pi=\left(i_{0}, b_{1}, i_{1}, \ldots, i_{n-1}, b_{n}, i_{n}\right)$ of bonds $b_{m} \in B\left(\mathbb{Z}^{d}\right), m=1, \ldots, n$ and of vertices $i_{m} \in \mathbb{Z}^{d}, m=0, \ldots, n$, such that $b_{m}=\left\{i_{m-1}, i_{m}\right\}$ for $m=1, \ldots, n$; the bonds $b_{i}$ are then said to belong to the path and sometimes a path is indicated by the list of its bonds only. Note that with our definition, both bonds and vertices can appear more than once; however, if $\left|\operatorname{Vert}\left(b_{m}\right) \cap \operatorname{Vert}\left(b_{l}\right)\right|=0$ when $|m-l| \neq 1$, then we say that the path is self-avoiding or, briefly, s.a.; note that sometimes the difference between self-avoiding or not might be essential, and we are careful in indicating when a path needs to be or must be self-avoiding. Given two paths $\pi_{1}=\left(b_{1}, \ldots, b_{n}\right)$ and $\pi_{2}=\left(c_{1}, \ldots, c_{m}\right)$, we indicate by $\left(\pi_{1}, \pi_{2}\right)$ the sequence $\left(b_{1}, \ldots, b_{n}, c_{1}, \ldots, c_{n}\right)$, and if such sequence, completed by an appropriate selection of vertices, is a path we call it the concatenation of the two paths. Next, we define a circuit to be a path $\pi=\left(i_{0}, b_{1}, i_{1}, \ldots, i_{n-1}, b_{n}, i_{n}\right)$ such that also $i_{0}=i_{n} ;$ a circuit $\pi=\left(b_{1}, \ldots, b_{n}\right)$ is s.a. if the path $\left(b_{1}, \ldots, b_{n-1}\right)$ is such. Finally, define a box to be a set of bonds $\Lambda$ such that $\operatorname{Vert}(\Lambda)=\left[k_{1}, h_{1}\right] \times\left[k_{2}, h_{2}\right] \times \ldots \times\left[k_{d}, h_{d}\right] \cap \mathbb{Z}^{d}$ for some $k_{i}, h_{i} \in \mathbb{Z}$, $k_{i}<h_{i}$. In particular, let $\Lambda_{k}$ be such that $\operatorname{Vert}\left(\Lambda_{k}\right)=[-k, k]^{d} \cap \mathbb{Z}^{d}$; for a box $\Lambda$ let $\partial \Lambda$ be the boundary of $\Lambda$, that is, the bonds of $\Lambda$, one end point of which belongs also to a bond not in $\Lambda$. Later, we consider sequences of random variables indexed by boxes and we always take the limit in the sense of Van Hove, that is, along sequences of boxes $\Lambda_{n_{k}}$ such that $\lim _{k \rightarrow \infty}\left|\partial \Lambda_{n_{k}}\right| /\left|\Lambda_{n_{k}}\right|=0$.

Next, we discuss configurations of interactions which, until Section 4 , are taken two-valued. Let

$$
J=\left\{J_{i, j}\right\}_{\substack{i, j \in \mathbb{Z}^{d} \\|i-j|=1}} \in\{-1,1\}^{B\left(\mathbb{Z}^{d}\right)}=\mathscr{J}
$$


be a given prescription of the interaction between vertices of $\mathbb{Z}^{d}$. We say that a set $E$ of bonds is $J$-frustrated, or is frustrated with respect to $J$, if and only if there is a circuit $\pi \subseteq E$ such that $\Pi_{b \in \pi} J_{b}=-1$; we also call such a circuit frustrated. If no frustrated circuit can be found, then $E$ is $J$-unfrustrated or is unfrustrated with respect to $J$. A set of vertices $V$ is then frustrated with respect to some $J$ if and only if the set of bonds $\{\{i, j\}, i, j \in V\}$ is $J$-frustrated.

Third, we identify subsets of $B\left(\mathbb{Z}^{d}\right)$ by configurations $\eta=\left\{\eta_{i j}\right\}_{i, j \in \mathbb{Z}^{d},|i-j|=1} \in$ $\{0,1\}^{B\left(\mathbb{Z}^{d}\right)}=H$, which we then call frustrated if there exists a circuit $\pi \subseteq$ $\eta^{-1}(1)$ such that $\Pi_{b \in \pi} J_{b}=-1$; here $\eta^{-1}(1)$ indicates the set of bonds in which the configuration $\eta$ takes value +1 (later, by a slight abuse of notation, it also indicates its cardinality). Given a configuration $\eta$ and a set $A \subset B\left(\mathbb{Z}^{d}\right)$, we indicate by $\eta_{A}$ the restriction of $\eta$ to $A$ and by $\eta_{\backslash A}$ the restriction of $\eta$ to $B\left(\mathbb{Z}^{d}\right) \backslash A$; sometimes, if $A \subset B(\Lambda)$, we use $\eta_{\backslash A}=\eta_{B(\Lambda) \backslash A}$ when no confusion can arise. We also identify the restriction $\eta_{A}$ of a configuration $\eta$ with the cylinder of configurations coinciding with $\eta_{A}$ on $A$. We say that a configuration $\eta$ is $J$-unfrustrated in a set of bonds $S$ if it is unfrustrated with respect to the restriction of $J$ to $\eta^{-1}(1)$; hence there is no circuit $\pi \subset S \cap \eta^{-1}(1)$ such that $\Pi_{b \in \pi} J_{b}=-1$. Given two sets $A, B \subset B\left(\mathbb{Z}^{d}\right)$, with $A \cap B=\varnothing$, and two configurations $\eta_{A}$ and $\eta_{B}$, we denote by $\eta_{A} \vee \eta_{B}$ the configuration $\eta_{A \cup B}$ which coincides with $\eta_{A}$ in $A$ and with $\eta_{B}$ in $B$; the same notation is used with $J$ replacing $\eta$.

Next, we give the definitions concerning percolation (see also [14]). We call a bond $b$ occupied (in $\eta$ ) if $\eta_{b}=1$, and a path $\pi$ occupied if all of its bonds $b \in \pi$ are occupied. Given $\eta$, we consider two vertices connected if there is an occupied path containing both vertices; then the set $\operatorname{Vert}\left(\eta^{-1}(1)\right)$ falls apart into maximal connected components called clusters. We say that percolation from the origin (or from a vertex $v$ ) occurs if $\operatorname{Vert}\left(\eta^{-1}(1)\right)$ contains at least one infinite cluster containing the origin (or the vertex $v$, respectively). On the other hand, we say that percolation occurs if $\operatorname{Vert}\left(\eta^{-1}(1)\right)$ contains at least one infinite cluster. Equivalent definitions are obtained as follows. Percolation from the origin is equivalent to the existence of an infinite occupied self-avoiding path $\pi$ containing the origin. Next, let $C_{v, \partial \Lambda}$ be the event that there is an occupied path $\pi$ whose set of vertices $\operatorname{Vert}(\pi)$ includes the vertex $v \in \operatorname{Vert}(\Lambda)$ and a vertex $w \in \operatorname{Vert}(\partial \Lambda)$. Denote by $C_{v, \infty}$ the existence of an infinite cluster including $v$, then $C_{v, \infty}=\cap_{k \geq k_{0}} C_{v, \partial \Lambda_{k}}$ for some $k_{0}$.

The sets of occupied bonds are described by a random mechanism, a simple form of which is obtained by defining, on the $\sigma$-algebra of $H$ generated by cylinders, the following Bernoulli probability measures conditioned to avoiding frustration. The motivation for this definition is that it captures relevant features of the more interesting FK random cluster measures (to be discussed later). Let $J \in \mathscr{J}$ and $p \in(0,1)$ and let $\Lambda=\Lambda_{k} \subset B\left(\mathbb{Z}^{d}\right)$ be a box. Given $\eta_{\Lambda}=\{0,1\}^{\Lambda} \in H_{\Lambda}$, let

$$
\nu_{J, p, \Lambda}\left(\eta_{\Lambda}\right)=\frac{p^{\eta_{\Lambda}^{-1}(1)}(1-p)^{\eta_{\Lambda}^{-1}(0)} U_{J}\left(\eta_{\Lambda}\right)}{Z_{J, p, \Lambda}},
$$


where $U_{J}\left(\eta_{\Lambda}\right)$ is the indicator function that $\eta_{\Lambda}$ is unfrustrated with respect to $J$, that is,

$$
U_{J}\left(\eta_{\Lambda}\right)= \begin{cases}1, & \text { if the set } \eta_{\Lambda}^{-1}(1) \text { is unfrustrated with respect to } J, \\ 0, & \text { otherwise }\end{cases}
$$

$Z_{J, p, \Lambda}$ is a normalizing factor, and $\eta_{\Lambda}^{-1}(i), i=0,1$, indicates, with a slight abuse of notation, the cardinality of the set $\eta_{\Lambda}^{-1}(i)$ on which $\eta$ takes the value $i$. Let $\nu_{J, p}$ be any weak limit of the sequence of probability measures $\nu_{J, p, \Lambda_{k}}$ taken as $k$ diverges to infinity, where $\Lambda_{k}$ is a sequence of boxes such that $\bigcup_{k} \Lambda_{k}=\mathbb{Z}^{d}$, and the limit is taken in the sense of Van Hove (as discussed above). We call every such measure a conditionally J-unfrustrated Bernoulli measure. We are interested in percolation under a measure $\nu_{J, p}$. There is no immediate equivalence, in this case, between occurrence of percolation from the origin with positive $\nu_{J, p}$ probability and occurrence of percolation with $\nu_{J, p}$-probability 1 ; therefore, we focus on the second type of phenomenon for the time being; a simple argument in Lemma 2.7 below shows that percolation a.e. implies percolation from the origin with $\nu_{J, p}$ positive probability. To simplify the discussion we introduce two critical points,

$$
\underline{p}_{c}(d)=\sup \left\{p: \forall J \quad \forall \nu_{J, p} \quad \nu_{J, p}(\text { percolation occurs })=0\right\}
$$

and

$$
\bar{p}_{c}(d)=\inf \left\{p: \forall J \quad \forall \nu_{J, p} \quad \nu_{J, p}(\text { percolation occurs })>0\right\} .
$$

This is just one of the possible definitions of critical points (see [24], page 39, for a related discussion and other possible definitions). We remark that, although we cannot determine, in general, monotonicity properties in $p$ of $\nu_{J, p}$ (percolation occurs) for fixed $J$, such monotonicity can be shown for the configuration of interactions $\bar{J}$ such that $\bar{J} \equiv 1$. In fact, in this case any measure $\nu_{\bar{J}, p, \Lambda}$, and thus any weak limit $\nu_{\bar{J}, p}$, is just a Bernoulli measure with occupation density $p$; monotonicity in $p$ of the probability that percolation occurs follows from the FKG inequality. This, in particular, implies that $\underline{p}_{c}(d) \leq \bar{p}_{c}(d)$.

A standard technique allows determining the absence of percolation in $\mathbb{Z}^{d}$ : if $p \leq p_{c}(d)$, the critical point for independent bond percolation, percolation does not occur for all $J$, that is, $\underline{p}_{c}(d) \geq p_{c}(d)$ (see [24], Proposition 3.6, for the proof of a more general statement). Actually, in the present case $\underline{p}_{c}(d)=p_{c}(d)$ (see Theorem 2.8 below).

It is a different matter to establish if for a given (sufficiently large) $p$, percolation occurs for all $J$. A positive answer is given in Theorem 2.2 below, which is preceded by some definitions and a technical lemma.

In order to discuss the simplest cases first, we now restrict to $\mathbb{Z}^{2}$, in which case $p_{c}(2)=1 / 2$ (see [17] and [18]).

The graph $\left(\mathbb{Z}^{2}, B\left(\mathbb{Z}^{2}\right)\right)$ has a dual graph, having set of vertices $\left(\frac{1}{2}, \frac{1}{2}\right)+\mathbb{Z}^{2}$ and bonds between all pairs of vertices at distance 1 . Each bond $b$ of the dual graph crosses a bond of $B\left(\mathbb{Z}^{2}\right)$ which is called its image $I(b)$ and $\gamma \subset B\left(\mathbb{Z}^{2}\right)$ is 
the image of a (self-avoiding) dual circuit if $I^{-1}(\gamma)$ is a (self-avoiding) circuit of the dual. Given a box $\Lambda \subset B\left(\mathbb{Z}^{2}\right)$, the image $\gamma \subset \Lambda$ of a self-avoiding dual circuit contained in $\Lambda$ separates $\Lambda \backslash \gamma$ into two parts, an inner part, $\operatorname{Int}_{\gamma}(\Lambda)$ say, and an outer part, $\operatorname{Est}_{\gamma}(\Lambda)$, in the sense that each path from $\operatorname{Int}_{\gamma}(\Lambda)$ to $\operatorname{Est}_{\gamma}(\Lambda)$ must intersect $\gamma$; moreover, $\partial \Lambda \subset \operatorname{Est}_{\gamma}(\Lambda) \cup \gamma$.

Next, fix $J \in \mathscr{J}$ and a box $\Lambda$. Let $\gamma$ be the image of a dual circuit and let $\eta_{\Lambda \backslash \gamma} \in\{0,1\}^{\Lambda \backslash \gamma}$ be a $J$-unfrustrated configuration in $\Lambda \backslash \gamma$. The next lemma states that given $J$ and $\eta_{\Lambda \backslash \gamma}$, there is at least one way of partitioning the bonds of $\gamma$ into at most two subsets such that all the bonds in each subset can be simultaneously occupied without generating frustration. Given a subset $A \subset \gamma$, define $\eta^{(A)}$ by

$$
\eta^{(A)}(b)= \begin{cases}\eta_{\Lambda \backslash \gamma}(b), & \text { if } b \notin \gamma \\ 1, & \text { if } b \in A, \\ 0, & \text { if } b \in \gamma \backslash A\end{cases}
$$

A set of bonds $A \subset \gamma$ is called conditionally frustrated if there exists a frustrated circuit $\pi \subset\left(\eta^{(A)}\right)^{-1}(1) ; A$ is called conditionally unfrustrated if there is no such circuit.

LEMMA 2.1. Given a box $\Lambda$, the image of a self-avoiding dual circuit $\gamma \subset \Lambda$, a configuration of interactions $J$ and a configuration $\eta_{\Lambda \backslash \gamma}$, J-unfrustrated in $\Lambda \backslash \gamma$, there exists at least one subset $A$ of $\gamma$ such that both $A$ and $\gamma \backslash A$ are conditionally unfrustrated.

PROOF. We recursively define subsets $A_{i} \subset \gamma$ by examining, in an arbitrary fixed order, the bonds $b_{1}, b_{2}, \ldots, b_{|\gamma|}$ of $\gamma$. Let $A_{1}=\left\{b_{1}\right\}$, which is obviously conditionally unfrustrated; then suppose that $A_{i-1}$ is conditionally unfrustrated and consider $b_{i}$. If $A_{i-1} \cup\left\{b_{i}\right\}$ is conditionally unfrustrated then let $A_{i}=A_{i-1} \cup\left\{b_{i}\right\}$; otherwise let $A_{i}=A_{i-1}$. By construction, $A=A_{|\gamma|}$ is conditionally unfrustrated, and the lemma is proved if $B=\gamma \backslash A_{|\gamma|}$ is also such. Suppose that it is not. Then there exists a frustrated circuit $\pi$ in $\left(\eta^{(B)}\right)^{-1}(1) ; \pi$ can be made self-avoiding by successively removing unfrustrated s.a. loops (i.e., circuits) or by taking the first s.a. loop if one is found to be frustrated. Then $\pi$ must necessarily satisfy $|\pi \cap \gamma|=k \geq 2$, with $k$ even. In fact, it is not possible that $\pi \subset \Lambda \backslash \gamma$, as $\eta_{\Lambda \backslash \gamma}$ is unfrustrated; therefore, $\pi$ must contain bonds of $\gamma$; as $\pi$ is self-avoiding, which is here an essential property, it must have nonempty intersection with both $\operatorname{Int}_{\gamma}(\Lambda)$ and $\operatorname{Est}_{\gamma}(\Lambda)$. Therefore, being a circuit, it must cross $\gamma$ an even number of times. We can write $\pi=\left(b_{i_{1}}, \pi_{i_{1}, i_{2}}, b_{i_{2}}, \pi_{i_{2}, i_{3}}, \ldots, \pi_{i_{(k-1)}, i_{k}}, b_{i_{k}}, \pi_{i_{k}, i_{1}}\right)$, where $b_{i_{j}} \in B$, and $\pi_{i_{j}, i_{(j+1)}} \subset \Lambda \backslash \gamma$ (here $i_{k+1}=i_{1}$ ) are s.a. paths; we can take $i_{k}>i_{j}$, for $j=1, \ldots, k-1$, in the above fixed order; moreover, $k$ is even. After examining $b_{i_{k}}$, each of the $b_{i_{j}}$ 's has been examined and not added to $A_{i_{j}}$, so that $A_{i_{j}-1} \cup\left\{b_{i_{j}}\right\}$ is conditionally frustrated. Thus there exists a frustrated circuit 
$\left(b_{i_{j}}, \pi_{j}\right) \subset \eta_{A_{i_{j}-1} \cup\left\{b_{i_{j}}\right\}}^{-1}(1) \subseteq \eta_{A \cup\left\{b_{i_{j}}\right\}}^{-1}(1)$, where $b_{i_{j}} \notin \pi_{j}$ and $\pi_{j} \subset \eta_{A}^{-1}(1)$. Next, form the circuit

$$
\tilde{\pi}=\left(\pi_{1}, \pi_{i_{1}, i_{2}}, \pi_{2}, \pi_{i_{2}, i_{3}}, \ldots, \pi_{k-1}, \pi_{i_{(k-1)}, i_{k}}, \pi_{k}, \pi_{i_{k}, i_{1}}\right),
$$

which can always be formed by traversing each $\pi_{j}$ in a suitably chosen direction, that is, by replacing $b_{i_{j}}$ by $\pi_{j}$ in $\pi$. Note that $\tilde{\pi} \subset \eta_{A}^{-1}(1)$ and let

$$
\tilde{\tilde{\pi}}=\left(b_{i_{1}}, \pi_{1}, \pi_{i_{1}, i_{2}}, b_{i_{2}}, b_{i_{2}}, \pi_{2}, \pi_{i_{2}, i_{3}}, \ldots, \pi_{k-1}, \pi_{i_{(k-1)}, i_{k}}, b_{i_{k}}, b_{i_{k}}, \pi_{k}, \pi_{i_{k}, i_{1}}, b_{i_{1}}\right) ;
$$

then

$$
\begin{aligned}
\prod_{b \in \tilde{\pi}} J_{b} & =\prod_{b \in \tilde{\tilde{\pi}}} J_{b} \\
& =\left(\prod_{j=1, \ldots, k} \prod_{b \in\left(b_{i_{j}}, \pi_{j}\right)} J_{b}\right) \times \prod_{b \in \pi} J_{b}=(-1)^{k+1}=-1,
\end{aligned}
$$

which contradicts the construction of $A$.

We now use one of the sets determined in Lemma 2.1 to show the occurrence of percolation for large enough $p$.

THEOREM 2.2. In $\mathbb{Z}^{2}$, for all $J \in\{-1,1\}$, if $p>8 / 9$ then percolation occurs $\nu_{J, p}$ a.e., that is, $\bar{p}_{c}(2) \leq 8 / 9$.

Proof. For a set $\gamma \in B\left(\mathbb{Z}^{2}\right)$, we indicate with $\gamma \equiv 0$ the event that $\eta_{b}=0$ for all $b \in \gamma$. If with $\nu_{J, p}$-probability 1 there are finitely many images of selfavoiding dual circuits $\gamma$ such that $\gamma \equiv 0$, then percolation occurs, as discussed below. Given the image $\gamma$ of a self-avoiding dual circuit and a box $\Lambda \supset \gamma$, Lemma 2.1 implies that for any configuration $\eta_{\Lambda \backslash \gamma}$ there exists a subset $A$ of $\gamma$ having the property that if none of the bonds of $\gamma \backslash A$ is occupied, then any subset of the bonds of $A$ can be occupied without creating frustration, and vice versa, exchanging the roles of $A$ and $\gamma \backslash A$. Denoting $|A|=m \in\{0, \ldots,|\gamma|\}$, we have for each $\eta_{\Lambda \backslash \gamma}$,

$$
\begin{aligned}
\nu_{J, p, \Lambda} & \left(\gamma \equiv 0 \mid \eta_{\Lambda \backslash \gamma}\right) \\
& =\frac{(1-p)^{|\gamma|}}{\sum_{\eta_{\gamma}: U_{J}\left(\eta_{\Lambda \backslash \gamma} \vee \eta_{\gamma}\right)=1} p^{\eta_{\gamma}^{-1}(1)}(1-p)^{\eta_{\gamma}^{-1}(0)}} \\
& \leq \frac{(1-p)^{|\gamma|}}{\sum_{k=0}^{m}\left(\begin{array}{c}
m \\
k
\end{array}\right) p^{k}(1-p)^{|\gamma|-k}+\sum_{k=0}^{|\gamma|-m}\left(\begin{array}{c}
|\gamma|-m \\
k
\end{array}\right) p^{k}(1-p)^{|\gamma|-k}} \\
& \leq \sup _{0 \leq m \leq|\gamma| / 2} \frac{(1-p)^{|\gamma|}}{(1-p)^{|\gamma|-m}+(1-p)^{m}} \\
& \leq \frac{1}{2}(1-p)^{|\gamma| / 2} .
\end{aligned}
$$


It follows that also $\nu_{J, p, \Lambda}(\gamma \equiv 0) \leq \frac{1}{2}(1-p)^{|\gamma| / 2}$ and taking $\Lambda \rightarrow \infty$ we get $\nu_{J, p}(\gamma \equiv 0) \leq \frac{1}{2}(1-p)^{|\gamma| / 2}$ for any weak limit $\nu_{J, p}$. For a given $k$, the number of images of self-avoiding dual circuits with exactly $k$ bonds containing the origin in their interior can easily be bounded by $k 3^{k}$. Then if $p>\frac{8}{9}$,

$$
\sum_{\gamma} \nu_{J, p}(\gamma \equiv 0) \leq \sum_{k \geq 1} k 3^{k} \frac{1}{2}(1-p)^{k / 2}<\infty
$$

by Borel-Cantelli, $\nu_{J, p}$ a.e. there are only finitely many images $\gamma$ of selfavoiding dual circuits surrounding the origin such that $\gamma \equiv 0$. Taking, for instance, a vertex external to all such $\gamma$ 's but in the boundary of at least one, then such vertex must be in an infinite cluster.

The question arises whether it is possible to close the gap between $1 / 2$ and $8 / 9$ and determine (at least the existence of) a value $p=\underline{p}_{c}=\bar{p}_{c}$. In Section 3 a negative answer is suggested, and proved for several graphs, by giving explicit positive lower bounds on $\underline{p}_{c}-\bar{p}_{c}$. In greater generality, but with no explicit bounds, the inequality $\underline{p}_{c}<\bar{p}_{c}$ is shown also in [5] and [7].

We now introduce the more interesting class of $\mathrm{FK}$ random cluster measures and discuss the extension of the above result to such measures and to general dimension $d$. To simplify the notation and unify the treatment of conditionally unfrustrated Bernoulli and FK random cluster measures, we introduce a parameter $q=1,2$ (which in the spin interpretation describes the number of possible spin values). The conditionally unfrustrated Bernoulli measure is included in the definition which follows by taking the (somewhat trivial) case of $q=1$, while $q=2$ correponds to the FK measures. Other values of $q$ are sometimes considered (such as integer $q \geq 3$ in the Potts model), but, for simplicity of exposition, we do not discuss these cases. We also give definitions in $\mathbb{Z}^{d}$, but simple modifications allow making the same construction for other regular graphs (such as the planar triangular graph which is also briefly discussed in Section 4 of this paper).

Let $q=1,2$ be fixed. Let $\Lambda=\Lambda_{k}$ be a box and let $\eta_{\Lambda} \in H_{\Lambda}=\{0,1\}^{\Lambda}$ and $\omega_{\Lambda} \in \Omega_{\Lambda}=\{1,3-2 q\}^{\operatorname{Vert}(\Lambda)}$; then define

$$
P_{J, \Lambda}^{(1)}\left(\eta_{\Lambda}, \omega_{\Lambda}\right)=\nu_{J, P, \Lambda}\left(\eta_{\Lambda}\right)
$$

and

$$
P_{J, \Lambda}^{(2)}\left(\eta_{\Lambda}, \omega_{\Lambda}\right)=\frac{p^{\eta_{\Lambda}^{-1}(1)}(1-p)^{\eta_{\Lambda}^{-1}(0)} 1_{\eta_{\Lambda} \sim \omega_{\Lambda}, J}}{Z_{J, \Lambda}},
$$

where $1_{\eta_{\Lambda} \sim \omega_{\Lambda}, J}$ equals 1 if we have $\omega_{\Lambda}(x) \omega_{\Lambda}(y) J_{\Lambda}(b)=1$ for each bond $b=$ $\{x, y\} \in \eta_{\Lambda}^{-1}(1)$, and equals 0 otherwise; and $Z_{J, \Lambda}$ is a normalizing factor. $P_{J, \Lambda}^{(q)}$ is a finite volume joint distribution of bond random variables $\eta_{\Lambda}$ and of spin variables $\omega_{\Lambda}$. We have not mentioned boundary conditions which are generally included in the definition (see, e.g., [24], pages 28-31), but our results are general enough to hold for all possible boundary conditions, and the required 
modifications from our presentation, which only discusses the case with no boundary conditions, are immediate.

The interesting measures are the marginals of $P_{J, \Lambda}^{(q)}$ and their weak limits. Given a configuration $\eta_{\Lambda} \in H_{\Lambda}$, the set $\eta_{\Lambda}^{-1}(1)$ defines, as before, connected components of the graph $(\operatorname{Vert}(\Lambda), \Lambda)$ and thus clusters; we then let $\operatorname{cl}\left(\eta_{\Lambda}\right)$ be the number of clusters. We then have

$$
\nu_{J, p, \Lambda}^{(q)}\left(\eta_{\Lambda}\right):=\sum_{\omega_{\Lambda} \in \Omega_{\Lambda}} P_{J, \Lambda}^{(q)}\left(\eta_{\Lambda}, \omega_{\Lambda}\right)=\frac{p^{\eta_{\Lambda}^{-1}(1)}(1-p)^{\eta_{\Lambda}^{-1}(0)} U_{J_{\Lambda}}\left(\eta_{\Lambda}\right) q^{\mathrm{cl}\left(\eta_{\Lambda}\right)}}{Z_{J, \Lambda}}
$$

and

$$
\mu_{J, \Lambda, \beta}\left(\omega_{\Lambda}\right):=\sum_{\eta_{\Lambda} \in H_{\Lambda}} P_{J, \Lambda}^{(q)}\left(\eta_{\Lambda}, \omega_{\Lambda}\right)=\frac{\exp \left(\beta \sum_{i, j \in \Lambda,|i-j|=1} J_{i j} \omega_{i} \omega_{j}\right)}{Z_{J, \Lambda}}
$$

where $U_{J_{\Lambda}}\left(\eta_{\Lambda}\right)$ equals 1 if $\eta_{\Lambda}^{-1}(1)$ is unfrustrated with respect to $J_{\Lambda}$ on the $\operatorname{graph}\{\operatorname{Vert}(\Lambda), \Lambda\}$, and equals 0 otherwise; $\beta$ is such that $p=1-e^{-2 \beta}, Z_{J, \Lambda}$ is used to indicate the appropriate normalizing factor, possibly different from one expression to the other and $\nu_{J, p, \Lambda}^{(1)}=\nu_{J, p, \Lambda}$ is as in (2.1) (see, e.g., [24], Propositions 3.2 and 3.2, for a proof). For $q=1$, the expression in (2.7) is trivial. For $q=2$, the measure in (2.7) is the finite volume Gibbs random field for the interaction $J$, and the measure in (2.6) is the finite volume $F K$ random cluster measure. We are then interested in the weak limits, as increasing subsequences of boxes $\Lambda$ cover $\mathbb{Z}^{d}$ in the sense of Van Hove, of the finite volume measures in (2.6) and (2.7), indicated hereafter by $\nu_{J, p}^{(q)}$. In particular, for $J \equiv 1$, that is, $J_{b}=1$ for all bond $b$, the measure (2.7) is the (finite volume) ferromagnetic Ising model with parameter $\beta$, where, in physical terms, $\beta$ represents the inverse temperature. Equation (2.6) is, instead, the finite volume ferromagnetic FK random cluster model studied in [10]; in such cases, it is shown in [24] that the weak limit of (2.6) is unique.

Let $\underline{p}_{c}^{(2)}(d)$ and $\bar{p}_{c}^{(2)}(d)$ be defined as in (2.2) and (2.3) for the measures $\nu_{J, p}^{(2)}$ (from now on we indicate $\underline{p}_{c}^{(1)}(d)=\underline{p}_{c}(d)$ and $\bar{p}_{c}^{(1)}(d)=\bar{p}_{c}(d)$ ) and let $\underline{p}_{c}^{(q)}(T)$ and $\bar{p}_{c}^{(q)}(T)$ be defined analogously for the triangular lattice. We re$\underline{p}_{c}^{c}$ mark that $\underline{p}_{c}^{(2)}(d) \leq \bar{p}_{c}^{(2)}(d)$ as seen again by taking $\bar{J}$ s.t. $\bar{J} \equiv 1$. In this case, the finite volume distributions are stochastically ordered (see [24]) and thus $\nu_{\bar{J}, p}^{(2)}$ (percolation occurs), where $\nu_{\bar{J}, p}^{(2)}$ is the unique weak limit of the finite volume distributions (2.6), is monotone increasing in $p$.

The next corollary extends the result of Theorem 2.2 to the FK random cluster measure in $\mathbb{Z}^{2}$.

COROLlaRY 2.3. In $\mathbb{Z}^{2}$, for all $J \in\{-1,1\}$, if $p>16 / 17$ then percolation occurs $\nu_{J, p}$ a.e., that is, $\bar{p}_{c}^{(2)}(2) \leq 16 / 17$. 
Proof. It is only required to modify (2.4), which now becomes

$$
\begin{aligned}
& \nu_{J, p, \Lambda}^{(2)}\left(\gamma \equiv 0 \mid \eta_{\Lambda \backslash \gamma}\right) \\
& \quad \leq \frac{(1-p)^{|\gamma|}}{\sum_{k=0}^{m}\left(\begin{array}{c}
m \\
k
\end{array}\right)(p / 2)^{k}(1-p)^{|\gamma|-k}+\sum_{k=0}^{|\gamma|-m}\left(\begin{array}{c}
|\gamma|-m \\
k
\end{array}\right)(p / 2)^{k}(1-p)^{|\gamma|-k}} \\
& \quad \leq \sup _{0 \leq m \leq|\gamma| / 2} \frac{(1-p)^{|\gamma|}}{(1-p)^{|\gamma|-m}(1-p / 2)^{m}+(1-p)^{m}(1-p / 2)^{|\gamma|-m}} \\
& \quad \leq \frac{1}{2}\left(\frac{2(1-p)}{2-p}\right)^{|\gamma| / 2},
\end{aligned}
$$

with $\gamma \subset \mathbb{Z}^{2}$ and $\eta_{\Lambda \backslash \gamma}$ as in (2.4), Therefore,

$$
\sum_{\gamma} \nu_{J, p}^{(2)}(\tilde{\gamma} \equiv 0) \leq \sum_{k} k 3^{k} \frac{1}{2}\left(\frac{2(1-p)}{2-p}\right)^{k / 2},
$$

which is bounded if $(2(1-p) /(2-p))^{1 / 2}<1 / 3$, that is, $p>16 / 17$.

We now consider the extension of the above results to higher dimensions $d$. We have two results, one relevant for large $d$ obtained by adapting [20], and the other useful for moderate dimensions, shown by comparison with dimension $d=2$.

We give some geometric notions that allow defining Peierls' contours, which constitutes the generalization of dual circuits. We first consider the dual lattice of $\mathbb{Z}^{d}$, which can be taken as $\mathbb{Z}^{d}+(1 / 2,1 / 2, \ldots, 1 / 2)$. A plaquette is then a unit $(d-1)$-dimensional cube from the dual lattice centered at the middle of some bond (when considering the bond as a line connecting $u$ to $v$ embedded in $\mathbb{R}^{d}$ ) of the initial lattice $\mathbb{Z}^{d}$. Two plaquettes are called adjacent if they have a common $(d-2)$-dimensional face, while, analogously, two bonds of the original lattice are called connected if they share a vertex. A set of plaquettes (or bonds) is called connected if any two of its elements belong to a chain of pairwise adjacent plaquettes (or bonds, respectively) from the set. As defined before, the boundary of a connected set $\Sigma$ of bonds is the set of all bonds sharing a vertex with a bond not in $\Sigma$. Let a connected set $\Sigma$ of bonds be given. For every bond $b$ in its boundary let us draw a plaquette orthogonal to $b$ and intersecting it in the middle point: the set of such plaquettes form closed surfaces; each connected component $\gamma$ is called Peierls' contour and the number of its plaquettes is indicated by $|\gamma|$. Each Peierls' contour $\gamma$ separates thus $\mathbb{R}^{d} \backslash \gamma$ into a bounded and an unbounded part, and a Peierls' contour containing the origin in the bounded part is called a Peierls' contour including the origin. Note that in $d=2$ Peierls' contour corresponds exactly to images of s.a. dual circuits. By taking as $\gamma$ a Peierls' contour, Lemma 2.1 can be extended by repeating its proof verbatim.

LEMMA 2.4. Given a box $\Lambda$, the image of a Peierls' contour $\gamma \subset \Lambda$, a configuration of interactions $J$ and a configuration $\eta_{\Lambda \backslash \gamma}, J$-unfrustrated in $\Lambda \backslash \gamma$, there 
exists at least one subset $A$ of $\gamma$ such that both $A$ and $\gamma \backslash A$ are conditionally unfrustrated.

It is shown in [20] that in $\mathbb{Z}^{d}$ the number of different Peierls' contours of size $n$ including the origin is less than $\exp [64 n(\log d) / d]$. Therefore, Theorem 2.2 and Corollary 2.3 can be adapted to deal with dimensions $d \geq 3$. The only change needed are in (2.5), which becomes

$$
\sum_{\gamma} \nu_{J, p}(\gamma \equiv 0) \leq \sum_{k \geq 1} \exp [64 k(\log d) / d] \frac{1}{2}(1-p)^{k / 2}<\infty
$$

and in (2.9), which becomes

$$
\sum_{\gamma} \nu_{J, p}(\gamma \equiv 0) \leq \sum_{k \geq 1} \exp [64 k(\log d) / d] \frac{1}{2}\left(\frac{2(1-p)}{2-p}\right)^{k / 2}<\infty .
$$

By determing sufficient conditions for convergence of these series we get the following result.

LEMMA 2.5. In $\mathbb{Z}^{d}$,

$$
\bar{p}_{c}^{(1)}(d) \leq 1-\exp [-128(\log d) / d]
$$

and

$$
\bar{p}_{c}^{(2)}(d) \leq \frac{2(1-\exp (-64(\log d) / d))}{2-\exp (-64(\log d) / d)}
$$

The next result is once again stated in $\mathbb{Z}^{d}$ for simplicity, but can easily be extended to any regular graph having a subgraph isomorphic to $\mathbb{Z}^{2}$ (see [19], page 10 , for related definitions). Let $\mathbb{Z}^{d}=\left\{\left(x_{1}, \ldots, x_{d}\right), x_{i} \in \mathbb{Z}\right\}$ and let $\tilde{\mathscr{I}}$ be the graph having as vertices the vertices of $\mathbb{Z}^{d}$ with $x_{d}=0$ and as bonds those bonds between them.

LEMMA 2.6. In $\mathbb{Z}^{d}, \bar{p}_{c}^{(1)}(d) \leq 8 / 9$ and $\bar{p}_{c}^{(2)}(d) \leq 16 / 17$.

Proof. The idea is to apply the reasoning leading to (2.4), (2.5) or (2.8), (2.9), respectively, to the bonds in $\tilde{\mathscr{G}}$ after conditioning to the configuration outside $\tilde{\mathscr{G}}$. Let $\gamma$ be a Peierls' contour of $\mathbb{Z}^{d}$ surrounding the origin $O$, and consider $\gamma \cap \tilde{\mathscr{G}}$, each connected component of which is the image of a s.a. dual circuit of $\mathbb{Z}^{2}$. We denote by $\tilde{\gamma}$ the one such component surrounding $O$. Next, denote by $S_{\gamma}$ the event that $\gamma \equiv 0$. If $S_{\gamma}$ occurs for only finitely many $\gamma$ 's then percolation occurs. Note that

$$
\nu_{J, p}\left(S_{\gamma}\right)=\lim _{\Lambda \rightarrow \infty} \nu_{J, p, \Lambda}\left(S_{\gamma}\right)
$$

and let $\tilde{\Lambda}=\Lambda \cap \tilde{\mathscr{G}}$; then $\nu_{J, p, \Lambda}\left(S_{\gamma}\right)=\sum_{\eta_{\Lambda \backslash \tilde{\Lambda}} \in H_{\Lambda \backslash \tilde{\Lambda}}} \nu_{J, p, \Lambda}\left(S_{\gamma} \mid \eta_{\Lambda \backslash \tilde{\Lambda}}\right) \nu_{J, p, \Lambda}\left(\eta_{\Lambda \backslash \tilde{\Lambda}}\right)$.

Given $\eta_{\Lambda \backslash \tilde{\Lambda}}$, if $S_{\gamma}$ occurs for some Peierls' contour $\gamma$ then $\tilde{\gamma}$ is such that

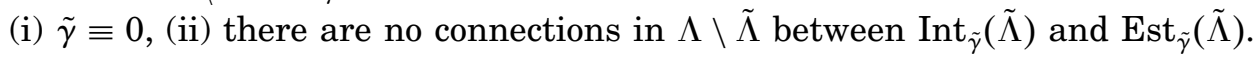


Note that if for all $\eta_{\Lambda \backslash \tilde{\Lambda}}$ the number of images $\tilde{\gamma}$ of s.a. dual circuits satisfying (i) and (ii) is bounded with $\nu_{J, p}$-probability 1 (on $\{0,1\}^{\tilde{\mathscr{G}}}$ ), then percolation occurs. Given $\eta_{\Lambda \backslash \tilde{\Lambda}}$, we denote the set of $\tilde{\gamma}$ such that (ii) occurs by $\tilde{\Gamma}_{\eta_{\Lambda \backslash \tilde{\Lambda}}}$ and for a given $\tilde{\gamma} \in \tilde{\Gamma}_{\eta_{\Lambda \backslash \tilde{\Lambda}}}$ by $S_{\tilde{\gamma}}$ the event that also (i) occurs. To each $\tilde{\gamma} \in \tilde{\Gamma}_{\eta_{\Lambda \backslash \tilde{\Lambda}}}$ Lemmas 2.1 and 2.4 apply since all possible connections between $\operatorname{Int}_{\tilde{\gamma}}(\tilde{\Lambda})$ and

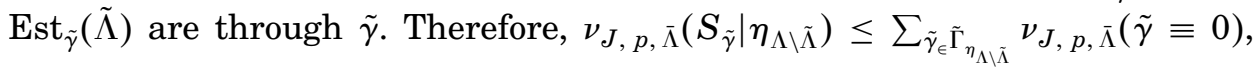
and, as in (2.5) or (2.9), for every $\eta_{\Lambda \backslash \tilde{\Lambda}}$,

$$
\nu_{J, p}\left(S_{\tilde{\gamma}} \text { occurs for some } \tilde{\gamma} \in \tilde{\Gamma}_{\eta_{\Lambda \backslash \tilde{\Lambda}}} \mid \eta_{\Lambda \backslash \tilde{\Lambda}}\right) \leq \sum_{k \geq 1} k 3^{k} \frac{1}{2}(1-p)^{|k| / 2}<\infty
$$

or

$$
\nu_{J, p}\left(S_{\tilde{\gamma}} \text { occurs for some } \tilde{\gamma} \in \tilde{\Gamma}_{\eta_{\Lambda \backslash \tilde{\Lambda}}} \mid \eta_{\Lambda \backslash \tilde{\Lambda}}\right) \leq \sum_{k \geq 1} k 3^{k} \frac{1}{2}\left(\frac{2(1-p)}{2-p}\right)^{|k| / 2}<\infty .
$$

The bound on the right-hand side is independent of $\Lambda$ and, taking $\Lambda \rightarrow \infty$, one gets that percolation occurs with $\nu_{J, p}$-probability 1 for $p<8 / 9$ or $16 / 17$, respectively.

The next lemma shows that the occurrence of percolation implies occurrence of percolation from the origin. To simplify the statement we indicate by percolation almost everywhere (a.e.) the occurrence of percolation with $\nu_{J, p^{-}}$ probability 1 and by percolation from the origin the occurrence of percolation from the origin with positive $\nu_{J, p}$-probability.

LEMMA 2.7. In the conditionally unfrustrated Bernoulli measure and in the $F K$ random cluster measure on $\mathbb{Z}^{d}$ percolation a.e. implies percolation from the origin.

Proof. Suppose percolation occurs with $\nu_{J, p}$-probability 1 . Then, by $\sigma$ additivity of the measure, there exists a vertex $v \in V$ such that percolation occurs from $v$ with positive $\nu_{J, p}$-probability. Select a path $\pi=\left(b_{1}, b_{2}, \ldots, b_{k}\right)$ from $v$ to the origin $O$ and a box $\Lambda \supset \pi$. Fix a configuration $\eta_{\Lambda \backslash \pi}$ and successively examine the bonds of $\pi$ recursively defining a set $A$. Consider $b_{1}=$ $\left\{v_{1,1}, v_{1,2}\right\}$; if $v_{1,1}$ and $v_{1,2}$ are already connected in $\eta_{\Lambda \backslash \pi}^{-1}(1)$ then $A_{1}=\varnothing$, otherwise let $A_{1}=\left\{b_{1}\right\}$. Define

$$
\eta^{(1)}(b)= \begin{cases}\eta_{\Lambda \backslash \pi}(b), & \text { if } b \notin \pi \\ 1, & \text { if } b \in A_{1}\end{cases}
$$

Suppose $b_{i-1}$ has been analyzed and $A_{i-1}$ has been defined, and consider $b_{i}=$ $\left\{v_{i, 1}, v_{i, 2}\right\}$. If $v_{i, 1}$ and $v_{i, 2}$ are already connected in $\left(\eta^{(i-1)}\right)^{-1}(1)$ then let $A_{i}=$ $A_{i-1}$, otherwise let $A_{i}=A_{i-1} \cup\left\{b_{i}\right\}$. Define

$$
\eta^{(i)}(b)= \begin{cases}\eta_{\Lambda \backslash \pi}(b), & \text { if } b \notin \pi \\ 1, & \text { if } b \in A_{i}\end{cases}
$$


The set $A\left(\eta_{(\Lambda \backslash \pi)}\right)=A=A_{k}$ has the property that if all the bonds in $A$ are occupied then $v$ is connected to all vertices in $\pi$; moreover, all the bonds in $A$ can be occupied simultaneously. Finally, since at each step only bonds between vertices not previously connected are added, the probability of such an occupation is at least $p^{|A|}$. Let $S_{O, v}$ be the event that $O$ is connected to $v$, let $S_{v, \partial \Lambda}$ be the event that there is a connection from $v$ to the boundary $\partial \Lambda$ of $\Lambda$ and let $S_{\pi, \partial \Lambda}$ be the event that there is an occupied path included in $\Lambda \backslash \pi$ from a vertex of $\pi$ to $\partial \Lambda$. Note that if $S_{v, \partial \Lambda}$ occurs, then either $v$ is connected to $\partial \Lambda$ by an occupied path included in $\Lambda \backslash \pi$, or a vertex of $\pi$ is connected to $\partial \Lambda$ by such path; this implies that if $\eta \in S_{v, \partial \Lambda}$ then $\eta_{\Lambda \backslash \pi} \in S_{\pi, \partial \Lambda}$, so that $\nu_{J, p}\left(S_{\pi, \partial \Lambda}\right) \geq \nu_{J, p}\left(S_{v, \partial \Lambda}\right)$ for every $J$ and every $p$. Since also $\nu_{J, p}\left(S_{v, \partial \Lambda}\right)>$ $\nu_{J, p}(v$ percolates $)$ we have

$$
\begin{aligned}
\nu_{J, p}\left(S_{O, v} \cap S_{v, \partial \Lambda}\right) & =\sum_{\eta_{\Lambda \backslash \pi} \subset S_{\pi, \partial \Lambda}} \nu_{J, p}\left(S_{O, v} \cap S_{v, \partial \Lambda} \mid \eta_{\Lambda \backslash \pi}\right) \nu_{J, p}\left(\eta_{\Lambda \backslash \pi}\right) \\
& \geq \sum_{\eta_{\Lambda \backslash \pi} \subset S_{\pi, \partial \Lambda}} p^{\left|A\left(\eta_{\Lambda \backslash \pi}\right)\right|} \nu_{J, p}\left(\eta_{\Lambda \backslash \pi}\right) \\
& \geq p^{|\pi|} \nu_{J, p}\left(S_{v, \partial \Lambda}\right) \\
& \geq p^{|\pi|} \nu_{J, p}(v \text { percolates })>0 .
\end{aligned}
$$

After taking the limit $\Lambda \rightarrow \infty$ this shows that also percolation from the origin occurs with positive $\nu_{J, p}$-probability.

From now on, since we always establish the occurrence of percolation a.e., by "occurrence of percolation" we indicate both occurrence of percolation from the origin of the lattice with positive probability and occurrence of percolation with probability 1 .

Let $p_{c}(d)$ be the critical value of independent bond percolation in $\mathbb{Z}^{d}, \beta_{c}(d)$ be the critical point of the $d$-dimensional Ising model and $\beta_{c}(T)=\frac{1}{4} \ln 3 \approx$ 0.276 be the critical point of the triangular lattice. Note that the exact value of $\beta_{c}(d)$ is known only in $d=2$, in which case it is $\beta_{c}(2)=\frac{1}{2} \ln (1+\sqrt{2})$ (see [4], page 77), and thus we have percolation for no $J$ if $p<1-1 / 1+\sqrt{2}=$ $2-\sqrt{2} \approx 0.586$. The following theorem collects all the results proved in the previous lemmas.

Only the statement (2.15) concerning the triangular lattice requires one additional modification, its proof being as the proof of Corollary 2.3 with $k 2^{k}$ replacing $k 3^{k}$ in (2.9).

THEOREM 2.8. Let $d \geq 2$. We have

$$
\underline{p}_{c}^{(1)}(d)=p_{c}(d)
$$

and

$$
\bar{p}_{c}^{(1)}(d) \leq \min \left\{\frac{8}{9}, 1-\exp (-128(\log d) / d)\right\}
$$


For the FK random cluster measure we have

$$
\underline{p}_{c}^{(2)}(d)=1-\exp \left(-2 \beta_{c}(d)\right)
$$

and

$$
\bar{p}_{c}^{(2)}(d) \leq \min \left\{\frac{16}{17}, \frac{2(1-\exp (-64(\log d) / d))}{2-\exp (-64(\log d) / d)}\right\} .
$$

Finally, for the FK random cluster measure on the triangular lattice $T$, defined as in (2.6) with $T$ replacing $\mathbb{Z}^{d}$, we have

$$
\underline{p}_{c}^{(2)}(T)=1-\exp \left(-2 \beta_{c}(T)\right)=1-\frac{1}{\sqrt{3}} \approx 0.423
$$

and

$$
\bar{p}_{c}^{(2)}(T) \leq \frac{6}{7} .
$$

3. Percolation for almost all interactions and strict inequalities. In this section we assume that the interactions are independent and randomly chosen according to a Bernoulli distribution $\mathscr{P}$ such that $\mathscr{P}\left(J_{b}=1\right)=\frac{1}{2}=$ $1-\mathscr{P}\left(J_{b}=-1\right)$ for all bonds $b$. Densities of ferromagnetic bonds other than $1 / 2$ can obviously be treated, but for simplicity we restrict the exposition to density $1 / 2$, which corresponds to the standard spin glass model. Having selected and fixed one such $J$, we want to study, as in the last section, the occurrence of percolation of occupied bonds at different values of the occupation probability $p$, with respect to a conditionally unfrustrated Bernoulli measure $\nu_{J, p}=\nu_{J, p}^{(1)}$ or to an FK random cluster measure $\nu_{J, p}^{(2)}$. The interesting feature is that, in general, there are values of $p$ such that percolation occurs for $\mathscr{P}$-almost all $J$ 's but does not occur for some $J$ 's.

To begin, we need to specify a map $J \rightarrow \nu_{J, p}^{(q)}$ which, to all, or at least $\mathscr{P}$ almost all, $J$ 's assigns one of the measures in which we are interested. By following and adapting [2], [23], [24], [25] and [26], one could determine a map which to $\mathscr{P}$-almost all $J$ 's assigns a distribution on the $\nu_{J, p}^{(q)}$, s with the physically interesting property of being translationally covariant. However, such a construction would become somewhat cumbersome and obscure some of our results. Therefore, we follow a less general but much simpler construction close to the one in [13].

For given $p$ and a box $\Lambda$ consider the joint distribution of $J_{\Lambda}$ and $\eta_{\Lambda}$ in $\Lambda$,

$$
P_{p, \Lambda}^{(q)}\left(J_{\Lambda}, \eta_{\Lambda}\right)=\mathscr{P}\left(J_{\Lambda}\right) \nu_{J_{\Lambda}, p, \Lambda}^{(q)}\left(\eta_{\Lambda}\right) \text {. }
$$

Let $P_{p}^{(q)}$ be any weak limit of $P_{p, \Lambda}^{(q)}$ along a suitable subsequence of boxes $\Lambda$ converging to $\mathbb{Z}^{d}$ (or, with suitable modifications, to $T$ ). Next, let $\nu_{J, p}^{(q)}=P_{p}^{(q)}(\cdot \mid J)$ be the conditional distribution of $\eta$ given $J$ and $P_{p \text {, edge }}^{(q)}$ be the marginal of $P_{p}^{(q)}$ on the $\eta$ variables. We adopt here a slight abuse of notation, since, for given $J$, the $\nu_{J, p}^{(q)}$ is not necessarily unique, nor does it necessarily coincide 
with those defined in Section 2 (in the first place, the current definition is well given for $\mathscr{P}$-almost all $J$ 's only). Nonetheless, the results of Section 2 hold for all $J$ 's and all weak limits $\nu_{J, p}^{(q)}$ however taken, and thus carry over to the possibly more restricted class of measures defined here.

The main result of this section concerns the occurrence or absence of percolation $\mathscr{P}$-almost everywhere and is based on extending Theorem 3 in [13]. We obtain upper and lower bounds, which hold for $\mathscr{P}$-almost all $J$ 's, for the conditional occupation probabilities. These bounds allow comparing our measures with the independent Bernoulli measure on the same graph, and then estimating the regions where percolation does or does not occur. The comparison between measures is based on the following inequality, already discussed in [14]: given two measures $\nu_{1}$ and $\nu_{2}$ on same graph $\mathscr{I}$, if for all $b \in \mathscr{G}$ and for all $\eta_{\backslash b}^{(1)}, \eta_{\backslash b}^{(2)} \in\{0,1\}^{\mathscr{C} \backslash b}$ we have $\nu_{1}\left(\eta_{b}=1 \mid \eta_{\backslash b}^{(1)}\right) \geq \nu_{2}\left(\eta_{b}=1 \mid \eta_{\backslash b}^{(2)}\right)$ then $\nu_{1}$ stochastically dominates $\nu_{2}$ (see [11]) and $\nu_{1}$ (percolation occurs) $\geq$ $\nu_{2}$ (percolation occurs).

LEMMA 3.1. For each bond $b$ in $\mathbb{Z}^{d}$ we have the following.

For $q=1$,

$$
\begin{aligned}
& \inf _{\eta_{\backslash b}} P_{p, \text { edge }}^{(1)}\left(\eta_{b}=1 \mid \eta_{\backslash b}\right) \geq \frac{p}{2}, \\
& \sup _{\eta_{\backslash b}} P_{p, \text { edge }}^{(1)}\left(\eta_{b}=1 \mid \eta_{\backslash b}\right) \leq p .
\end{aligned}
$$

This implies that

if $p>2 p_{c}(d)$ percolation occurs

for the conditional distribution $\nu_{J, p}^{(1)}$ for $\mathscr{P}$-almost all $J$,

if $p<p_{c}(d)$ percolation does not occur

for the conditional distribution $\nu_{J, p}^{(1)}$ for $\mathscr{P}$-almost all $J$.

For $q=2$,

$$
\begin{gathered}
\inf _{\eta_{\backslash b}} P_{p, \text { edge }}^{(2)}\left(\eta_{b}=1 \mid \eta_{\backslash b}\right) \geq \frac{p}{2}, \\
\sup _{\eta_{\backslash b}} P_{p, \text { edge }}^{(2)}\left(\eta_{b}=1 \mid \eta_{\backslash b}\right) \leq \frac{p}{2-2 p+p^{2}} .
\end{gathered}
$$

This implies that

$$
\text { if } p>2 p_{c}(d) \text { percolation occurs }
$$

for the conditional distribution $\nu_{J, p}^{(2)}$ for $\mathscr{P}$-almost all $J$,

$$
\text { if } p<\left(1+2 p_{c}(d)-\sqrt{1+4 p_{c}(d)-4\left(p_{c}(d)\right)^{2}}\right) / 2 p_{c}(d)
$$

percolation does not occur for the conditional distribution

$\nu_{J, p}^{(2)}$ for $\mathscr{P}$-almost all $J$. 
The same results as in (3.5)-(3.8) hold for the triangular lattice with $p_{c}(T)$ replacing $p_{c}(d)$.

These values must be compared with those of Theorem 2.8. In particular, for $q=1$, (3.4) does not say anything new with respect to (2.10); (3.3) is useless for $d=2$ where $p_{c}(d)=\frac{1}{2}$ but suggests that percolation for almost all $J$ occurs for the triangular lattice in a region potentially larger than that where percolation occurs for all $J$. For the FK random cluster model related to spin glasses, obtained for $q=2$, notice the following. In $d=2$, since $p_{c}(2)=1 / 2$, (3.8) holds, surprisingly, exactly the same bound of $2-\sqrt{2}$ as (2.2), although the two methods seem to be completely unrelated and (3.8) is only an upper bound. For other values of $d$ it is not easy to compare (3.8) and (2.2). However, we see that in the triangular lattice, where (3.8) gives about 0.520 and (2.14) about 0.423 , there does exist a new region. For $p$ between those two values percolation occurs for $\mathscr{P}$-almost no $J$, but, as the value in (2.14) is exactly the one above which there is percolation for $\bar{J}$ (with a $\bar{J} \equiv 1$ ), it does occur for some $J$. By improving upon (3.8), we see in Theorem 3.2 below that also in $\mathbb{Z}^{2}$ such a phenomenon occurs; on the other hand, we expect that in general there are also values of $p$ for which percolation occurs for $\mathscr{P}$-almost all $J$ but does not for some $J$. An example in which both phenomena occur is obtained, however, only for a particular distribution on $J$ (no longer two-valued) in Theorem 4.2 below.

PROOF. Taking a sequence of boxes $\Lambda_{n}$ along which the joint distribution $P_{\alpha, p, \Lambda_{n}}^{(q)}$ converges we have, by the martingale convergence theorem, that

$$
P_{\alpha, p, \text { edge }}^{(q)}\left(\eta_{b}=1 \mid \eta_{\backslash b}\right)=\lim _{\Lambda_{n} \rightarrow \infty} \frac{\sum_{J_{\Lambda_{n}}} \lim _{n} P_{\alpha, p, \Lambda_{n}}^{(q)}\left(\eta_{b} \vee \eta_{\Lambda_{n} \backslash b}, J_{\Lambda_{n}}\right)}{\sum_{\eta_{b}} \sum_{J_{\Lambda_{n}}} \lim _{n} P_{\alpha, p, \Lambda_{n}}^{(q)}\left(\eta_{b} \vee \eta_{\Lambda_{n} \backslash b}, J_{\Lambda_{n}}\right)} ;
$$

therefore, upper and lower bounds on

$$
\frac{\sum_{J_{b}} P_{\alpha, p, \Lambda_{n}}^{(q)}\left(\eta_{b} \vee \eta_{\Lambda_{n} \backslash b}, J_{\Lambda_{n}}\right)}{\sum_{J_{b}} \sum_{\eta_{b}} P_{\alpha, p, \Lambda_{n}}^{(q)}\left(\eta_{b} \vee \eta_{\Lambda_{n} \backslash b}, J_{\Lambda_{n}}\right)},
$$

which are independent from $J_{\backslash b}$ and $\eta_{\backslash b}$ are also bounds for the conditional probabilities $P_{\alpha, p \text {, edge }}^{(q)}\left(\eta_{b}=1 \mid \eta_{\backslash b}\right)$.

Using next the inequality mentioned before the statement of the lemma, it is possible to show that the marginal distribution on the $\eta$ 's is dominated or dominates a Bernoulli measure, so that, for appropriate choices of $p$, percolation occurs with probability zero or one. An application of Fubini's theorem yields that such a result holds also for the marginal $\nu_{J, p}^{(q)}$ for $\mathscr{P}$-almost all $J$ (see [13] for further details).

We now estimate the r.h.s. of (3.9) from below. Given $b=\{x, y\}, \eta_{\Lambda \backslash b}$ and $J_{\Lambda \backslash b}$, three situations can occur: either $x$ and $y$ are connected in $\eta_{\Lambda \backslash b}$, and in such a case this connection is ferromagnetic or antiferromagnetic, or $x$ and $y$ 
are not connected in $\eta_{\Lambda \backslash b}$. It is easy to see that after reducing the common factors, the conditional probabilities in the three cases are bounded as follows (see [13] for details). For $q=1,2$,

$$
\begin{aligned}
P_{p, \text { edge }}^{(q)}\left(\eta_{b}=1 \mid\right. & \left.\eta_{\Lambda \backslash b}, J_{\Lambda \backslash b}\right) \\
\geq \inf _{J} \min \left\{\frac{p / Z_{a}}{p / Z_{a}+(1-p)\left(1 / Z_{a}+1 / Z_{f}\right)},\right. & \left.\frac{p / Z_{f}}{p / Z_{f}+(1-p)\left(1 / Z_{a}+1 / Z_{f}\right)}, \frac{p / q}{p / q+(1-p)}\right\},
\end{aligned}
$$

where $Z_{f}\left(Z_{a}\right)=Z_{J, p, \Lambda}$ when $J=J_{\backslash b} \vee\left(J_{b}=1\right)\left(J=J_{\backslash b} \vee\left(J_{b}=-1\right)\right)$. The ratio $Z_{f} / Z_{a}$ remains to be estimated, but this can be done by taking inf and sup over $\eta_{\Lambda \backslash b}$ of the ratios of corresponding terms. Again, three situations can occur, giving rise to the following estimate:

$$
\begin{aligned}
1-p & =\min \left\{(1-p), \frac{1}{1-p}, 1\right\} \leq \frac{Z_{a}}{Z_{f}} \\
& \leq \max \left\{(1-p), \frac{1}{1-p}, 1\right\}=\frac{1}{1-p} ;
\end{aligned}
$$

therefore,

$$
P_{p, \text { edge }}^{(q)}\left(\eta_{b}=1 \mid \eta_{\Lambda \backslash b}, J_{\Lambda \backslash b}\right) \geq \min \left\{\frac{p}{2}, \frac{p / q}{p / q+(1-p)}\right\}=\frac{p}{2} .
$$

To prove (3.2) and (3.6) we repeat the previous steps, reversing the inequalities. In particular, $P_{p \text {, edge }}^{(q)}$ is bounded by taking the sup in the r.h.s. of (3.11), with $Z_{a} / Z_{f}$ satisfying (3.12). Therefore,

$$
\begin{aligned}
& P_{p, \text { edge }}^{(1)}\left(\eta_{b}=1 \mid \eta_{\Lambda \backslash b}, J_{\Lambda \backslash b}\right) \\
& \quad \leq \max \left\{\frac{p}{p+(1-p)(2-p)}, p /(p+q(1-p))\right\} .
\end{aligned}
$$

The corresponding statements for the triangular lattice are obtained by obvious substitutions.

A small improvement upon (3.2), and upon (3.6), can be obtained by a better estimate of the ratio $Z_{a} / Z_{f}$ than that given in (3.12). Such an improvement is really relevant only in $\mathbb{Z}^{2}$, where it shows the existence of $p \in(0,1)$ such that percolation occurs for $\mathscr{P}$-almost no $J$ but it does occur for some $J$. Although the improvement we obtain is very small, this result has some relevance since it proves that there is a strict inequality in the behavior of some quantities (here the probability of percolation) between the Ising model and spin glasses; altough widely expected, there were no rigorous proofs of any such strict inequality (recently, more general but less explicit inequalities have been discussed in [5] and [7]). 
THEOREM 3.2. Consider an FK random cluster model in $\mathbb{Z}^{2}$ with distribution $\mathscr{P}$ on $\mathscr{J}$. If $x \approx 0.588$ equals the unique root in $[0,1]$ of the equation

$$
4-4 x-18 x^{2}+39 x^{3}-37 x^{4}+17 x^{5}-3 x^{6}=0
$$

then for $p \in(2-\sqrt{2}, x)$ percolation occurs for $\mathscr{P}$-almost no $J$ but it does occur for some $J$.

PROOF. As seen in Lemma 3.1, $2-\sqrt{2}$ is the critical point for percolation if $J=\bar{J} \equiv 1$, so that for $p>2-\sqrt{2}$ percolation occurs for $\bar{J}$.

To show that it does not occur for $P$-almost all $J$ we follow the proof of (3.6). All steps are the same apart from the estimate of the ratio $Z_{a} / Z_{f}$. To get a better estimate, we use a small set around the given bond $b$ and take sums over $\eta$ by separating the indices in this set. In details, let $b=\{(-1,0),(0,0)\}$ be the given bond and let $\Lambda=\{b,\{(0,0),(1,0)\},\{(0,0),(0,1)\},\{(0,0),(0,-1)\}\}$. In the following formula we denote joint configurations such as $J_{A} \vee J_{B}$ simply by $\left(J_{A}, J_{B}\right)$, and $U_{J}(\eta)$ by $U(\eta, J)$ for typographical reasons; furthermore, a single number in the indication of the configuration $J$ indicates the value taken by $J_{b}$ for the bond $b$ under consideration. Given $\Lambda^{\prime} \supset \Lambda$ and $J_{\Lambda^{\prime} \backslash b}$, if a configuration $\eta_{\Lambda^{\prime} \backslash \Lambda}$ is unfrustated in $\Lambda^{\prime} \backslash \Lambda$ with respect to the restriction $J_{\Lambda^{\prime} \backslash \Lambda}$ of $J_{\Lambda^{\prime}}$ to $\Lambda^{\prime} \backslash \Lambda$ (i.e., if $U_{J_{\Lambda^{\prime} \backslash \Lambda}}\left(\eta_{\Lambda^{\prime} \backslash \Lambda}\right)=1$ ) we write $\eta_{\Lambda^{\prime} \backslash \Lambda} \approx J_{\Lambda^{\prime}}$. Then we have

$$
\begin{aligned}
Z_{a} / Z_{f} & =\frac{\sum_{\eta_{\Lambda^{\prime}}} p^{\eta_{\Lambda^{\prime}}^{-1}(1)}(1-p)^{\eta_{\Lambda^{\prime}}^{-1}(0)} 2^{\operatorname{cl}\left(\eta_{\Lambda^{\prime}}\right)} U\left(\eta_{\Lambda^{\prime}},\left(-1, J_{\Lambda^{\prime} \backslash b}\right)\right)}{\sum_{\eta_{\Lambda^{\prime}}} p^{\eta_{\Lambda^{\prime}}^{-1}(1)}(1-p)^{\eta_{\Lambda^{\prime}}^{-1}(0)} 2^{\operatorname{cl}\left(\eta_{\Lambda^{\prime}}\right)} U\left(\eta_{\Lambda^{\prime}},\left(1, J_{\Lambda^{\prime} \backslash b}\right)\right)} \\
& \geq \min _{\eta_{\Lambda^{\prime} \backslash \Lambda}: \eta_{\Lambda^{\prime} \backslash \Lambda} \approx J_{\Lambda^{\prime}}} \frac{\sum_{\eta_{\Lambda}} p^{\eta_{\Lambda^{\prime}}^{-1}(1)}(1-p)^{\eta_{\Lambda^{\prime}}^{-1}(0)} 2^{\operatorname{cl}\left(\eta_{\Lambda^{\prime}}\right.} U\left(\eta_{\Lambda^{\prime}},\left(-1, J_{\Lambda^{\prime} \backslash b}\right)\right)}{\sum_{\eta_{\Lambda}} p^{\eta_{\Lambda^{\prime}}^{-1}(1)}(1-p)^{\eta_{\Lambda^{\prime}}^{-1}(0)} 2^{\operatorname{cl}\left(\eta_{\Lambda^{\prime}}\right)} U\left(\eta_{\Lambda^{\prime}},\left(1, J_{\Lambda^{\prime} \backslash b}\right)\right)} .
\end{aligned}
$$

Given $J_{\Lambda^{\prime} \backslash \Lambda}$ and $\eta_{\Lambda^{\prime} \backslash \Lambda}$, such that $U\left(\eta_{\Lambda^{\prime} \backslash \Lambda}, J_{\Lambda^{\prime} \backslash \Lambda}\right)=1$, and given $J_{\Lambda \backslash b}$, the configurations of $H_{\Lambda \backslash b}$ can be classified, regardless of $J_{b}$, as follows. Let

$$
\begin{aligned}
\bar{H}= & \left\{\eta_{\Lambda \backslash b} \in H_{\Lambda \backslash b} \mid U\left(\eta_{b}=0, \eta_{\Lambda \backslash b}, \eta_{\Lambda^{\prime} \backslash \Lambda} ; J_{\Lambda \backslash b}, J_{\Lambda^{\prime} \backslash \Lambda}\right)=1\right\}, \\
H_{1}= & \left\{\eta_{\Lambda \backslash b} \in \bar{H} \mid \operatorname{both} U\left(\eta_{b}=1, \eta_{\Lambda \backslash b}, \eta_{\Lambda^{\prime} \backslash \Lambda} ; J_{b}=1, J_{\Lambda \backslash b}, J_{\Lambda^{\prime} \backslash \Lambda}\right)=1\right. \\
& \text { and } \left.U\left(\eta_{b}=1, \eta_{\Lambda \backslash b}, \eta_{\Lambda^{\prime} \backslash \Lambda} ; J_{b}=-1, J_{\Lambda \backslash b}, J_{\Lambda^{\prime} \backslash \Lambda}\right)=1\right\}, \\
H_{f}= & \left\{\eta_{\Lambda \backslash b} \in \bar{H} \mid \operatorname{both} U\left(\eta_{b}=1, \eta_{\Lambda \backslash b}, \eta_{\Lambda^{\prime} \backslash \Lambda} ; J_{b}=1, J_{\Lambda \backslash b}, J_{\Lambda^{\prime} \backslash \Lambda}\right)=1\right. \\
& \text { and } \left.U\left(\eta_{b}=1, \eta_{\Lambda \backslash b}, \eta_{\Lambda^{\prime} \backslash \Lambda} ; J_{b}=-1, J_{\Lambda \backslash b}, J_{\Lambda^{\prime} \backslash \Lambda}\right)=0\right\}, \\
H_{a}= & \left\{\eta_{\Lambda \backslash b} \in \bar{H} \mid \operatorname{both} U\left(\eta_{b}=1, \eta_{\Lambda \backslash b}, \eta_{\Lambda^{\prime} \backslash \Lambda} ; J_{b}=1, J_{\Lambda \backslash b}, J_{\Lambda^{\prime} \backslash \Lambda}\right)=0\right. \\
& \text { and } \left.U\left(\eta_{b}=1, \eta_{\Lambda \backslash b}, \eta_{\Lambda^{\prime} \backslash \Lambda} ; J_{b}=-1, J_{\Lambda \backslash b}, J_{\Lambda^{\prime} \backslash \Lambda}\right)=1\right\} .
\end{aligned}
$$

Let also

$$
H_{f}^{0}\left(H_{a}^{0}\right)=\left\{\eta_{\Lambda} \in H_{\Lambda}: \eta_{\Lambda \backslash b} \in H_{f}\left(H_{a}\right) \text { and } \eta_{b}=0\right\}
$$

and

$$
H_{f}^{1}\left(H_{a}^{1}\right)=\left\{\eta_{1} \in H_{\Lambda}: \eta_{\Lambda \backslash b} \in H_{f}\left(H_{a}\right) \text { and } \eta_{b}=1\right\}
$$


Note that $\bar{H}=H_{1} \cup H_{f} \cup H_{a}$, and that only the $\eta_{\Lambda}$ 's such that $\eta_{\Lambda \backslash b} \in \bar{H}$ give a nonzero contribution to the sums in (3.16).

If $\eta_{\Lambda \backslash b} \in H_{1}$, then both terms $\eta=\left(\eta_{b}=1, \eta_{\Lambda \backslash b}\right)$ and $\eta=\left(\eta_{b}=0, \eta_{\Lambda \backslash b}\right)$ give a nonzero contribution to both numerator and denominator of the last expression in (3.16). We consider separately all of these terms except $\underline{\eta}_{\Lambda \backslash b}$, which is the configuration such that $\underline{\eta}_{\Lambda \backslash b} \equiv 0$; note that, after collecting a term which is kept fixed from now on, $\left(\underline{\eta}_{\Lambda \backslash b}, \eta_{b}=1\right)$ contributes $p(1-p)^{3} / 2$ to the sums in (3.16) and $\left(\eta_{\Lambda \backslash b}, \eta_{b}=0\right)$ contributes $(1-p)^{4}$. Next, let $a(p)=$ $p(1-p)^{3} / 2+(1-p)^{4}$. If $\left(\eta_{\Lambda \backslash b}, \eta_{b}=1\right) \in H_{a}^{1}$ then it gives a nonzero contribution only to the numerator of the last term in (3.16), and we obtain a lower bound by removing it. We thus get from (3.16), indicating $\eta_{\Lambda}=\eta_{\Lambda \backslash b} \vee \eta_{b}$, and indicating 2 to the power of the number of different clusters of $\Lambda^{\prime}$ intersecting $\Lambda$ divided by $2^{5}$ as $2^{\text {cl }\left(\eta_{\Lambda}\right)} \geq 1$,

$$
\begin{aligned}
Z_{a} / Z_{f} & \\
\geq \min _{\eta_{\Lambda^{\prime} \backslash \Lambda} \approx J_{\Lambda^{\prime}}} \min ( & \min _{\eta_{\Lambda \backslash b} \in H_{1}, \eta_{\Lambda \backslash b} \neq \underline{\eta}_{\Lambda \backslash b}} \frac{\sum_{\eta_{b}} p^{\eta_{\Lambda}^{-1}(1)}(1-p)^{\eta_{\Lambda}^{-1}(0)} 2^{\operatorname{cl}\left(\eta_{\Lambda^{\prime}}\right)}}{\sum_{\eta_{b}} p^{\eta_{\Lambda}^{-1}(1)}(1-p)^{\eta_{\Lambda}^{-1}(0)} 2^{\operatorname{cl}\left(\eta_{\Lambda^{\prime}}\right)}}, \\
& \left.\frac{\sum_{\eta_{\Lambda} \in H_{f}^{0} \cup H_{a}^{0}} p^{\eta_{\Lambda \mid b}^{-1}(1)}(1-p)^{\eta_{\Lambda \backslash b}^{-1}(0)+1} 2^{\operatorname{cl}\left(\eta_{b}=0, \eta_{\Lambda \backslash b}\right)}+a(p)}{\sum_{\eta_{\Lambda} \in H_{f}^{0} \cup H_{a}^{0} \cup H_{f}^{1}} p^{\eta_{\Lambda}^{-1}(1)}(1-p)^{\eta_{\Lambda}^{-1}(0)} 2^{\operatorname{cl}\left(\eta_{\Lambda}\right)}+a(p)}\right) .
\end{aligned}
$$

So we have the following:

$$
\geq \min _{\eta_{\Lambda^{\prime} \backslash \Lambda} \approx J_{\Lambda^{\prime}}} \min \left(1, \frac{1}{1+\left(\frac{(1-p)}{p}+\frac{a(p)}{\sum_{\eta_{\Lambda} \in H_{f}^{1}} p^{\eta_{\Lambda}^{-1}(1)}(1-p)^{\eta_{\Lambda}^{-1}(0)} 2^{\mathrm{cl}\left(\eta_{\Lambda}\right)}}\right)^{-1}}\right),
$$

where the last inequality is obtained by disregading $H_{a}^{0}$ and observing that the ratio between the sums in $H_{f}^{0}$ and $H_{f}^{1}$ is $(1-p) / p$. By observing that $\sum_{\eta_{\Lambda} \in H_{f}^{1}} p^{\eta_{\Lambda}^{-1}(1)}(1-p)^{\eta_{\Lambda}^{-1}(0)} 2^{\mathrm{cl}\left(\eta_{\Lambda}\right)} \leq 1$ we get, from (3.1),

$$
\begin{aligned}
Z_{a} / Z_{f} & \geq \min \left(1,\left(1+\left(\frac{(1-p)}{p}+a(p)\right)^{-1}\right)^{-1}\right) \\
& =\frac{2-2 p+p(1-p)^{3}(2-p)}{2+p(1-p)^{3}(2-p)}=: L(p) \leq 1 .
\end{aligned}
$$

Exchanging the roles of $Z_{a}$ and $Z_{f}$, one can see that this is also a lower bound for $Z_{f} / Z_{a}$. Therefore, from the r.h.s. of (3.11) and from (3.19) we get

$$
\begin{aligned}
P_{\alpha, p, \text { edge }}^{(2)}\left(\eta_{b}=1 \mid \eta_{\Lambda^{\prime} \backslash b}, J_{\Lambda^{\prime} \backslash b}\right) & \leq \sup \left(\frac{p}{p+(1-p)(1+L(p))}, \frac{p}{p+2(1-p)}\right) \\
& =\frac{p}{p+(1-p)(1+L(p))} .
\end{aligned}
$$


Percolation does not occur if $p$ is such that $p /(p+(1-p)(1+L(p)))<1 / 2$, and since $p /(p+(1-p)(1+L(p)))$ is increasing, this is equivalent to saying that $p$ is less than the root $x$ of $x(x+(1-x) L(x))^{-1}=1 / 2$, that is, (3.15).

4. Other values of the interaction. In the previous sections the interaction $J$ assumed only the values 1 and -1 , but slightly different phenomena occur when $J$ takes other values, as discussed in this section. First, we need to briefly redefine all the measures with which we are dealing to take into account the other values of $J$; the main difference is that now at $p$ close to 1 the set $S$ of bonds which has the tendency to be occupied is, among the unfrustrated sets, the one which maximizes $\sum_{b \in S}\left|J_{b}\right|$. As a consequence, we need some care in extending Theorem 2.2, which is based on occupying at least half of the bonds of a given circuit. In redefining the model, the value of $p$, which is related to the (conditional) probability that a bond $b$ is open, must itself be influenced by the value of $J_{b}$; frustration, on the other hand, still depends only on $\operatorname{sign}\left(J_{b}\right)$. To allow the value $J_{b}=0$, a reasonable convention is that in this case the bond $b$ cannot be occupied; we include this in the definition of $U_{J}$. We consider $\mathbb{Z}^{d}$, as before, and let $I \subset \mathbb{R}$ be the set of possible values of the interaction. Let $\mathscr{J}=I^{B}, \phi_{J, p, \Lambda, 1}=\prod_{b \in \Lambda: \eta_{\Lambda}(b)=1}\left(1-(1-p)^{\left|J_{b}\right|}\right)$, $\phi_{J, p, \Lambda, 0}=\prod_{b \in \Lambda: \eta_{\Lambda}(b)=0}(1-p)^{\left|J_{b}\right|}$ and define for finite $\Lambda \subset B$ and $q=1,2$,

$$
\nu_{J, p, \Lambda}^{(q)}\left(\eta_{\Lambda}\right)=\frac{\phi_{J, p, \Lambda, 1} \phi_{J, p, \Lambda, 0} U_{J}\left(\eta_{\Lambda}\right) q^{\operatorname{cl}\left(\eta_{\Lambda}\right)}}{Z_{J, p, \Lambda}},
$$

where $U_{J}\left(\eta_{\Lambda}\right)$ is the indicator function that $\eta_{b}=0$ if $J_{b}=0, \eta_{\Lambda}$ is not frustrated (in the sense used before) with respect to the configuration $\operatorname{sign}(J) \in$ $\{-1,1\}$ defined, if $J_{b} \neq 0$, by $(\operatorname{sign}(J))_{b}=\operatorname{sign}\left(J_{b}\right)$, and $Z_{J, p, \Lambda}$ is the partition function.

As before, $\nu_{J, p, \Lambda}^{(q)}$ can be realized as marginal of a joint measure on $\eta$ 's and $\omega$ 's which is a conditional Bernoulli measure, and we omit these details now. The definition (4.1) and in particular the form of the factors $(1-p)^{\left|J_{b}\right|}$ and $1-(1-p)^{\left|J_{b}\right|}$ derive from the interpretation of (4.1) as a way of defining spin glass models. In this case, in fact, $p_{J_{b}}=1-\exp \left(-2 \beta\left|J_{b}\right|\right)$, and if $\left|J_{b}\right|=1$ we have $p=p_{1}=1-e^{-2 \beta}$; the form used in (4.1) expresses $p_{J_{b}}$ as a function of $p=p_{1}$. Of course, if $I=\{-1,1\}$, we obtain the models used in the previous sections.

Let $\mathscr{P}$, a distribution on $\mathscr{J}$, be a product of independent identical distributions on $I$. Then one can repeat the construction of Section 3 starting from (4.1), to obtain a joint distribution $P_{p}^{(q)}$ on $\mathscr{J} \times \mathscr{H}$.

Some of the methods of Section 2 apply if the distribution of $J$ is bounded away from 0 . As an example, we have the following.

LEMMA 4.1. For any fixed dimension $d$, suppose there exists $t>0$ such that for every bond $b$ we have $\left|J_{b}\right| \geq t$. Then for all $J$, percolation of occupied bonds does occur if $p>1-1 /\left((8 q+1)^{1 / t}\right)$ in the conditionally unfrustrated 
Bernoulli model and in the FK random cluster model on $\mathbb{Z}^{d}$, that is, $\bar{p}_{c}^{(q)}(d) \leq$ $1-1 /\left((8 q+1)^{1 / t}\right)$. In the triangular lattice, $\bar{p}_{c}(T) \leq 1-1 / 7^{1 / t}$.

Proof. Repeating the proof of Lemma 2.6 one gets, for $q=1,2$,

$$
\begin{aligned}
\nu_{J, p, \Lambda}\left(\tilde{\gamma} \equiv 0 \mid \eta_{\Lambda \backslash \tilde{\gamma}}\right) \leq & \prod_{b \in \tilde{\gamma}}(1-p)^{\left|J_{b}\right|} \\
& \left.\times\left[\sum_{\eta_{\tilde{\gamma}}: U_{J}\left(\eta_{\Lambda \backslash \tilde{\gamma}} \vee \eta_{\tilde{\gamma}}\right)=1} \sum_{b \in \tilde{\gamma}: \eta_{\tilde{\gamma}}(b)=1} \frac{\left(1-(1-p)^{\left|J_{b}\right|}\right)}{q} \times \prod_{b \in \tilde{\gamma}: \eta_{\tilde{\gamma}}(b)=0}(1-p)^{\left|J_{b}\right|}\right)\right]^{-1} \\
\leq & \left(\prod_{b \in A} \frac{\left(1+(q-1)(1-p)^{\left|J_{b}\right|}\right)}{q(1-p)^{\left|J_{b}\right|}}\right) \\
& \left.+\left(\prod_{b \in \tilde{\gamma} \backslash A} \frac{\left(1+(q-1)(1-p)^{\left|J_{b}\right|}\right)}{q(1-p)^{\left|J_{b}\right|}}\right)\right)^{-1} \\
\leq & \sup _{0 \leq m \leq \tilde{\gamma} \mid / 2} \frac{1}{\left(\frac{1+(q-1)(1-p)^{t}}{q(1-p)^{t}}\right)^{m}+\left(\frac{1+(q-1)(1-p)^{t}}{q(1-p)^{t}}\right)^{|\tilde{\gamma}|-m}} \\
\leq & \frac{q}{2}\left(\frac{q(1-p)^{t}}{1+(q-1)(1-p)^{t}}\right)^{|\tilde{\gamma}| / 2},
\end{aligned}
$$

where the second inequality is realized for some particular $A \subseteq \tilde{\gamma}$ chosen as in (2.4), that is, according to Lemma 2.1; the last inequality follows from the monotonicity of $x \rightarrow\left(1+(q-1)(1-p)^{x}\right) / q(1-p)^{x}$ so that any value of $\left|J_{b}\right|$ can be replaced by $t$. Then, if $3\left(q(1-p)^{t} / 1+(q-1)(1-p)^{t}\right)^{1 / 2}<1$, which is $(8 q+1)(1-p)^{t}<1$, that is, $p>1-1 /\left((8 q+1)^{1 / t}\right)$, we have

$$
\sum_{\tilde{\gamma}} \nu_{J, p, \Lambda}(\gamma \equiv 0) \leq \sum_{k \geq 1} k 3^{k} \frac{1}{2}\left(\frac{q(1-p)^{t}}{1+(q-1)(1-p)^{t}}\right)^{k / 2}<\infty .
$$

Lemma 2.7 applies, since it is based on frustration, that is, on $\operatorname{sign}(J)$ only, and thus percolation both a.e. and from the origin occurs. The result for the triangular lattice is obtained by replacing $3^{k}$ by $2^{k}$ in (4.3).

The next result shows that, for a particular graph and a particular choice of the values assumed by $J$, it is possible to identify four regions of the interval $(0,1)$ such that if $p$ is in these regions, percolation occurs for none, almost none, almost all or all of the $J$ 's, respectively. We cannot determine whether there is a sharp transition between the second and the third region as, somewhat surprisingly, a proof that the occurrence of percolation is monotone in $p$ is not available. In any case, we believe that the separation of the four regions, including sharp transition points, holds for a wide selection of graphs and of 
distributions of the J's. We show the result for the FK random cluster model in the triangular lattice.

THEOREM 4.2. Let $T$ be the triangular lattice, let $\delta \in(0,1)$ and $t>0$ be real numbers and let $J=\left\{J_{b}\right\}_{b \in T}$ be i.i.d. random variables distributed according to $\mathscr{P}$ with $\mathscr{P}(J=1)=\mathscr{P}(J=-1)=(1-\delta) / 2$ and $\mathscr{P}(J=t)=\mathscr{P}(J=-t)=$ $\delta / 2$. Given $\varepsilon \in(0,1 / 7)$, it is possible to take $\delta$ and $t$ small enough that for the $F K$ random cluster model on $T$ the following happens:

$$
\begin{aligned}
& \text { if } p<1-\exp \left(-2 \beta_{c}(T)\right)=1-1 / \sqrt{3} \approx 0.422 \text { percolation } \\
& \text { occurs for no } \mathrm{J},
\end{aligned}
$$

$$
\text { if } 1-1 / \sqrt{3}<p<\left(1+2 p_{c}(T)-\sqrt{1+4 p_{c}(T)-4\left(p_{c}(T)\right)^{2}}\right) /
$$
$2 p_{c}(T) \approx 0.452$, percolation occurs for some but $\mathscr{P}$-almost none of the J's,

if $1-1 / 7+\varepsilon<p<1-1 /(3)^{1 / 2 t}$ percolation occurs for $\mathscr{P}$-almost all but not all of the J's,

$$
\text { if } 1-1 / 7^{1 / t}<p \text { percolation occurs for all J's. }
$$

Here $\beta_{c}(T)$ and $p_{c}(T)$ are the critical points, on $T$, of the Ising model and of independent percolation, respectively.

PROOF. Equation (4.4) is shown by comparing the finite volume FK random cluster measure $\nu_{J, p, \Lambda}$ relative to each $J$ with that of $\bar{J}$, where $\bar{J}_{b}=1$ for all $b \in T$. In fact, $\nu_{J, p, \Lambda}$ is dominated by $\nu_{\bar{J}, p, \Lambda}$ for all $J$ (see (3.29) in [24]). By the exact solution of the Ising model on $T$, we get, as in (2.14), that if $p<1-\exp \left(-2 \beta_{c}(T)\right)=1-1 / \sqrt{3}$, then for no $J$ percolation occurs, while if $p>1-1 / \sqrt{3}$ then percolation occurs at least for $\bar{J}$; this shows (4.4) and part of (4.5).

To complete the proof of (4.5) we only need to show that, for $p$ in the given interval, percolation occurs for almost no $J$. We proceed as in showing (3.2) and (3.6), just modifying the calculations in (3.14) and (3.12).

By some calculation, it is possible to give an upper bound to $P_{p \text {, edge }}^{(2)}\left(\eta_{b} \mid \eta_{\Lambda \backslash b}\right.$, $J_{\Lambda \backslash b}$ ) in the form of (3.14), in which the maximum is taken among the same terms as in (3.14) plus additional terms for the case $J_{b}=t,-t$ involving the new normalization functions $Z_{t, a}$ and $Z_{t, f}$. For these it holds that

$$
\inf \left\{(1-p)^{t},(1-p)^{-t}, 1\right\} \leq Z_{t, a} / Z_{t, f} \leq \sup \left\{(1-p)^{t},(1-p)^{-t}, 1\right\} .
$$

Inserting these bounds in the above-mentioned upper bound, one can obtain

$$
\begin{aligned}
& P_{p, \text { edge }}^{(2)}\left(\eta_{b}=1 \mid \eta_{\Lambda \backslash b}, J_{\Lambda \backslash b}\right) \\
& \leq \sup \left\{\frac{p}{2-2 p+p^{2}}, \frac{1-(1-p)^{t}}{1-(1-p)^{t}+(1-p)^{t}\left(1+(1-p)^{t}\right)},\right. \\
& \left.p /(2-p), \frac{1-(1-p)^{t}}{1+(1-p)^{t}}\right\}
\end{aligned}
$$


all terms of which are, as $t$ is taken small, bounded again by $p /\left(2-2 p+p^{2}\right)$ as when $J$ takes only values \pm 1 , so that the bound (3.6) remains the same.

To show (4.6) we first find a configuration of $J$ for which percolation occurs exactly if and only if $p>1-1 /(\sqrt{3})^{1 / t}$ : this is done by taking $\tilde{J}$ such that $\tilde{J}_{b}=t$ for all $b \in T$. For this configuration of the interactions, one can refer again to the exact solution of the Ising model on the triangular lattice, just with $p$ replaced by $1-(1-p)^{t}$; the condition for percolation is thus $1-(1-p)^{t}>$ $1-1 / \sqrt{3}$, so that percolation does not occur for all $J$ if $p<1-1 / 3^{1 / 2 t}$. The lower bound in (4.6) is obtained by showing that for $\delta$ small the presence of bonds with $J_{b}= \pm t$ does not much influence the estimate in (2.8) used to show (2.15). In fact, by an estimate from the theory of greedy lattice animals in [9], the maximal fraction of bonds with $J_{b}= \pm t$ in a self-avoiding path near the origin is asymptotically bounded by any power of $\delta=P(|J|=t)$ smaller than $1 / 2$ with $\mathscr{P}$-probability 1 , in the sense that for every $c>0$ there exists a constant $h>0$ such that

$$
\lim _{n \rightarrow \infty} \frac{1}{n} \quad \max _{\pi: \pi \text { is a s.a. chain, }|\pi|=n, \pi \subseteq \Lambda_{n}} \sum_{b \in \pi} 1_{\left\{J_{b}= \pm t\right\}} \leq h \delta^{1 /(2+c)}
$$

$\mathscr{P}$-almost everywhere. The same bound applies to images of dual circuits surrounding the origin, since if they are of size $n$ they must lie within $\Lambda_{n}$. Therefore, we can repeat the proof of (2.15) by modifying the estimate as follows. Let $\gamma$ be the image of a dual circuit and let now $\tilde{\gamma}=\left\{b \in \gamma: J_{b}= \pm 1\right\}$ and $\gamma_{t}=\gamma \backslash \tilde{\gamma}$. Then, we can obtain a bound for $\nu_{J, p, \Lambda}^{(2)}(\gamma \equiv 0)$ using the r.h.s. of (2.18). Given the estimate on the size of $\tilde{\gamma}$, we get, by eliminating from the sum all terms with $\eta_{\gamma_{t}}^{-1}(1) \neq 0, \nu_{J, p, \Lambda}^{(2)}\left(\gamma \equiv 0 \mid \eta_{\Lambda \backslash \gamma}\right) \leq \frac{1}{2}(2(1-p) /(2-p))^{|\gamma|\left(1-h \delta^{1 /(2+c)}\right) / 2}$. Let $\gamma$ indicate images of s.a. circuit surrounding the origin; then, if $\delta$ is small enough,

$$
\sum_{\gamma} \nu_{J, p}(\gamma \equiv 0) \leq \sum_{k \geq 1} k 2^{k-1}(2(1-p) /(2-p))^{k\left(1-h \delta^{1 /(2+c)}\right) / 2}
$$

for $\mathscr{P}$-almost all $J$, since $k 2^{k-1}$ is an upper bound of the number of dual circuits in the triangular lattice; the r.h.s. of (4.8) is bounded if $p>1-1 / 7+\varepsilon$ for $\varepsilon$ small enough, so that percolation occurs as required. If $\varepsilon$ and $t$ are small enough, then $6 / 7+\varepsilon<1-1 /(\sqrt{3})^{1 / t}$ and (4.6) holds.

Finally, (4.7) is proved in Lemma 4.1.

The next theorem discusses the occurrence of percolation when $J$ can take the value 0 . Recall that bonds $b$ in which $J_{b}=0$ cannot be occupied, so that in this case we can only refer to percolation a.e., as the origin might be surrounded by bonds with $J_{b}=0$. Theorem 4.3 considers an FK random cluster model on $\mathbb{Z}^{d}$ (or on $T$ ). There exists $p$ large enough that percolation occurs, if and only if the density of nonzero interactions is strictly above the percolation threshold $p_{c}(d)$ [or $p_{c}(T)$ for $T$ ].

THEOREM 4.3. Consider a conditionally unfrustrated Bernoulli model or an $F K$ random cluster model on $\mathbb{Z}^{d}, d \geq 2$ (or on the triangular lattice $T$ ). There 
exists $p<1$ such that percolation occurs for $\mathscr{P}$-almost all $J$ if and only if $\mathscr{P}\left(J_{b} \neq 0\right)>p_{c}(d)\left[\right.$ or $p_{c}(T)$, respectively].

PROOF. We give the proof for $\mathbb{Z}^{d}$, which can be easily adapted to the triangular lattice.

If $p<1$ and $P\left(J_{b} \neq 0\right) \leq p_{c}(d)$, then the conditional probability of occupation of a bond $b$ is strictly less than $p_{c}(d)$, so that the joint distribution is dominated by a nonpercolating Bernoulli measure. An explicit estimate is given by selecting $M>0$ such that $P(|J|<M \mid J \neq 0)=1 / 2$ and computing, for $q=1,2$,

$$
\begin{aligned}
& P_{p, \text { edge }}^{(q)}\left(\eta_{b}=1 \mid \eta_{\backslash b}\right) \\
& \quad=P_{p}^{(q)}\left(\eta_{b}=1, J_{b} \neq 0 \mid \eta_{\backslash b}\right) \\
& \quad \leq\left(\frac{1}{2}+\frac{1}{2}\left(\frac{1-(1-p)^{M}}{1-(1-p)^{M}+q(1-p)^{M}}\right)\right) P\left(J_{b} \neq 0\right)<p_{c}(d) .
\end{aligned}
$$

If $\mathscr{P}\left(J_{b} \neq 0\right)>p_{c}(d)$, we want to rescale the $J$ variables: a schematic representation of the rescaled variables is shown in Figure 1. There exists

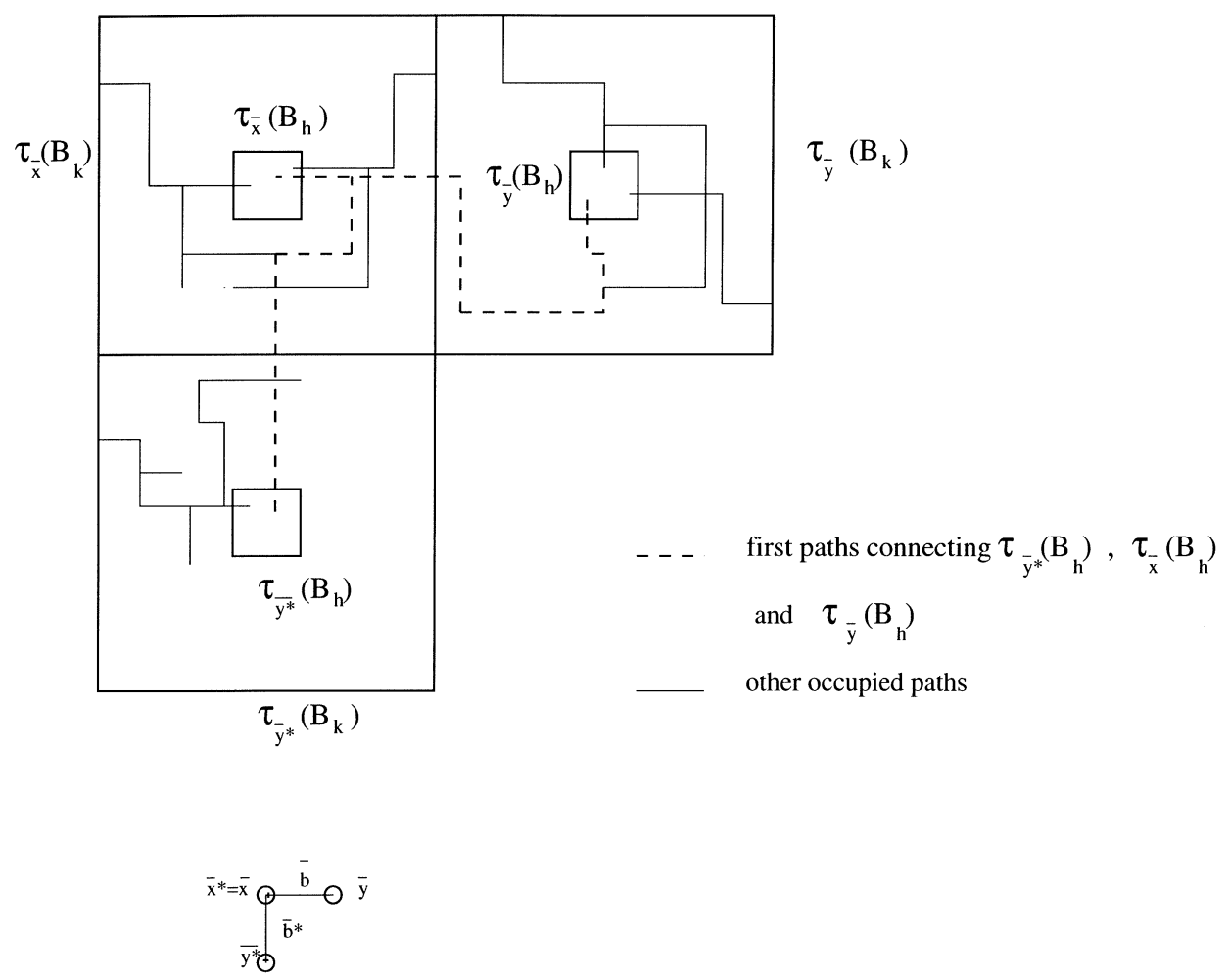

FIG. 1. 
$\delta>0$ such that $\mathscr{P}\left(J_{b} \notin[-\delta, \delta]\right)>p_{c}(d)$, and we focus on the bonds in which $J_{b} \notin[-\delta, \delta]$ for one such $\delta$ which is fixed from now on. For $h \in \mathbb{N}$, define the box $B_{h}=[-h, h]^{d} \cap \mathbb{Z}^{d}$. Let $k>h$, with $k, h \in \mathbb{N}$, and for $\bar{x} \in \mathbb{Z}^{d}$ let $\tau_{\bar{x}}=\tau_{2 k \bar{x}}$ denote, by a slight abuse of notation, the translation by $2 k$ times the vector $\bar{x}$; for $\bar{b}=(\bar{x}, \bar{y}) \in B\left(\mathbb{Z}^{d}\right)$ define the rescaled variable $\Xi_{\bar{b}} \in$ $\{0,1\}$ to be such that $\Xi_{\bar{b}}=1$ if and only if the following occurs. There is a path in $J^{-1}(\mathbb{R} \backslash[-\delta, \delta]) \cap\left(\tau_{\bar{x}}\left(B_{k}\right) \cup \tau_{\bar{y}}\left(B_{k}\right)\right)$ connecting $\tau_{\bar{x}}\left(B_{h}\right)$ to $\tau_{\bar{y}}\left(B_{h}\right)$, and, furthermore, all paths connecting $\partial \tau_{\bar{z}}\left(B_{h}\right)$ to $\partial \tau_{\bar{z}}\left(B_{k}\right)$ are connected to each other within $J^{-1}(\mathbb{R} \backslash[-\delta, \delta]) \cap \tau_{\bar{z}}\left(B_{k}\right)$, both for $\bar{z}=\bar{x}$ and $\bar{z}=\bar{y}$. If $\Xi_{\bar{b}}=1$ we say that $\Xi_{\bar{b}}$ occurs. Following the results in [15], the construction in [1] and uniqueness of the infinite cluster in independent percolation, it is possible to find, for every $\varepsilon>0$, suitable integers $k$ and $h$ such that for all $\bar{b} \in B\left(\mathbb{Z}^{d}\right)$ $P\left(\Xi_{\bar{b}}=1\right)>1-\varepsilon$. From now on we focus on the renormalized bonds $\bar{b} \in \mathbb{Z}^{2}$, which correspond to a layer $\mathbb{Z}^{2} \times[-k, k]^{d-2}$ in the original lattice; renormalized quantities are identified by decorating them with a bar.

The second step is to observe that the renormalized block variables $\Xi_{\bar{b}_{1}}$ and $\Xi_{\bar{b}_{2}}$ are independent if $\bar{b}_{1}$ and $\bar{b}_{2}$ have no common end point, that is, they are one-dependent. By [22], for any given Bernoulli 0-1 variables on the edges $B\left(\mathbb{Z}^{2}\right)$ of $\mathbb{Z}^{2}$ there exists $\varepsilon$ such that if $P\left(\Xi_{\bar{b}}=1\right)>\varepsilon$ for every $\bar{b} \in B\left(\mathbb{Z}^{2}\right)$, then the variables $\left\{\Xi_{\bar{b}}\right\}_{\bar{b} \in B\left(\mathbb{Z}^{2}\right)}$ stochastically dominate the given Bernoulli variables. For Bernoulli variables with occupation probability $p>$ $8 / 9$, we have that the probability that there exists the image $\gamma$ of a dual circuit surrounding the origin such that $|\{b: \gamma(b)=0\}|>1 / 2$ is less than $\sum_{k} k 3^{k-1}(1-p)^{k / 2}$, which is a bounded series. Therefore, it is possible to select $k$ and $h$ such that, with $\mathscr{P}$-probability 1 , all large images of dual circuits $\bar{\gamma}$ surrounding the origin contain at least $|\bar{\gamma}| / 8$ bonds $\bar{b}$ such that $\Xi_{\bar{b}}=1$.

Next choose an order of the bonds in each $\tau_{\bar{x}}\left(B_{h}\right), \bar{x} \in \mathbb{Z}^{2}$. For a given renormalized bond $\bar{b}=\{\bar{x}, \bar{y}\} \in \mathbb{Z}^{2}$, if $\Xi_{\bar{b}}$ occurs then there is a first bond $b_{1}$ in $\tau_{\bar{x}}\left(B_{h}\right)$ which is connected to $\tau_{\bar{y}}\left(B_{h}\right)$, and a first $b_{2}$ in $\tau_{\bar{y}}\left(B_{h}\right)$ connected to $b_{1}$ in $\tau_{\bar{x}}\left(B_{h}\right)$. Among all s.a. paths in $\left.J^{-1}[-\delta, \delta]\right)$ connecting $b_{1}$ and $b_{2}$ one can select the one which comes lexicographically first. Note that if $\Xi_{\bar{b}^{*}}$ occurs for some $\bar{b}^{*}=\left\{\bar{x}^{*}, \bar{y}^{*}\right\}$ sharing an end point with $\bar{b}$, say $\tau_{\bar{x}}\left(B_{h}\right)=\tau_{\bar{x}^{*}}\left(B_{h}\right)$, then the first bond in $\tau_{\bar{x}}\left(B_{h}\right)$ is the same: in fact, each bond which is reached by connections between $\tau_{\bar{x}}\left(B_{h}\right)$ and $\tau_{\bar{y}}\left(B_{h}\right)$ is also reached by connections between $\tau_{\bar{x}^{*}}\left(B_{h}\right)$ and $\tau_{\bar{y}^{*}}\left(B_{h}\right)$ as all s.a. paths from $\partial \tau_{\bar{x}^{*}}\left(B_{h}\right)$ and $\partial \tau_{\bar{x}^{*}}\left(B_{k}\right)$ are connected to each other.

For a given configuration of interactions $J$ let

$$
\begin{gathered}
R_{J}=\bigcup_{\bar{b}: \Xi_{\bar{b}} \text { occurs }}\left\{\pi_{\bar{b}}: \pi_{\bar{b}} \text { is the first s.a. path connecting } b_{1} \in\right. \\
\left.\tau_{\bar{x}}\left(B_{h}\right) \text { to } b_{2} \in \bar{\tau}_{\bar{y}}\left(B_{h}\right)\right\} .
\end{gathered}
$$

It is on $R_{J}$ that we find a percolation cluster of occupied bonds. In fact, let now $\eta_{\mathbb{Z}^{d} \backslash R_{J}}$ be a configuration such that percolation of occupied bonds does not occur in $\mathbb{Z}^{d} \backslash R_{J}$. If for some $\eta_{R_{J}}$ percolation does not occur in $\eta_{\mathbb{Z}^{d} \backslash R_{J}} \vee \eta_{R_{J}}$ 
then there exists the image of a dual hypersurface $\gamma$ surrounding the box $B_{h}$ such that $\gamma \equiv 0$. However, this implies that there exists the image of a dual self-avoiding circuit $\bar{\gamma}$ in $\mathbb{Z}^{2}$, seen as reference space of the block variables, such that $\bar{b}$ is not active for all the $\bar{b}$ in $\bar{\gamma}$ for which $\Xi_{\bar{b}}$ occurs; we indicate this event by saying that $\bar{\gamma}$ is not active. Given $J, \eta_{\mathbb{Z}^{d} \backslash R_{J}}$ and $\bar{b}=\{\bar{x}, \bar{y}\}$ such that $\Xi_{\bar{b}}$ occurs, it is possible to classify $\bar{b}$ as follows: either all connections which can be realized between $b_{1}$ and $b_{2}$ are of a given sign (ferromagnetic or antiferromagnetic) or both signs can be realized; this last case is actually the easiest to deal with as it can satisfy both constraints.

Given $\bar{\gamma}, \eta_{\mathbb{Z}^{d} \backslash R_{J}}$ and $\eta_{R_{J} \backslash \cup_{\bar{b} \bar{\gamma} \bar{\gamma}} \pi_{\bar{b}}}$, it is possible to apply Lemma 2.1 following the lines of Lemma 4.1. To estimate the probability that $\bar{\gamma}$ is not active, we can assume that on $\bar{\gamma}$ there are at least $|\bar{\gamma}| / 2$ bonds $\bar{b}$ for which $\Xi_{\bar{b}}$ occurs (as this does not happen only on a finite number of such $\bar{\gamma}$ and this is not relevant in our estimate). It is now possible to find a $J$-unfrustrated configuration $\eta_{R_{J}}$ in which at least $\bar{\gamma} / 4$ of the bonds of $\bar{\gamma}$ are such that $\Xi_{\bar{b}}$ occurs and $b_{1}$ and $b_{2}$ are connected (without creating frustration).

Applying the methods used in Lemma 4.1, one can obtain the estimate

$$
\begin{aligned}
& \nu_{J, p, \Lambda}\left(\bar{\gamma} \text { is not active } \mid \eta_{\Lambda \backslash R_{J}}\right) \\
& =\frac{\sum_{\mathscr{R}} \Pi_{b \in \eta_{R_{J, \Lambda}}^{-1}(1)}\left(1-(1-p)^{\left|J_{b}\right|}\right) \Pi_{b \in \eta_{R_{J, \Lambda}}^{-1}(0)}(1-p)^{\left|J_{b}\right|} q^{\mathrm{cl}\left(\eta_{\Lambda \backslash R_{J}}, \eta_{R_{J, \Lambda}}\right)}}{\sum_{\mathscr{Q}} \Pi_{b \in \eta_{R_{J, \Lambda}}^{-1}(1)}\left(1-(1-p)^{\left|J_{b}\right|}\right) \Pi_{b \in \eta_{R_{J, \Lambda}}^{-1}(0)}(1-p)^{\left|J_{b}\right|} q^{\mathrm{cl}\left(\eta_{\Lambda \backslash R_{J}}, \eta_{R_{J, \Lambda}}\right)}} \\
& \leq\left(\frac{2^{k_{0}} q(1-p)^{\delta}}{\left(1+(q-1)(1-p)^{\delta}\right)^{k_{0}}}\right)^{[|\bar{\gamma}| / 4]}
\end{aligned}
$$

where $k_{0}=(2 k+1)^{d}, R_{J, \Lambda}=R_{J} \cap \Lambda, \mathscr{U}=\left\{\eta_{R_{J, \Lambda}}: U_{J}\left(\eta_{R_{J, \Lambda}}, \eta_{\Lambda \backslash R_{J}}\right)=1\right\}$, and $\mathscr{R}=\left\{\eta_{R_{J, \Lambda}} \in \mathscr{U}: \bar{\gamma}\right.$ is not active in $\left.\eta_{R_{J, \Lambda}} \vee \eta_{\Lambda \backslash R_{J}}\right\}$. Now take $p$ large enough that $\left(2^{k_{0}} q(1-p)^{\delta} /\left(1+(q-1)(1-p)^{\delta}\right)^{k_{0}}\right)^{1 / 4}<(1 / 3)$. Either with $\nu_{J, p}$ positive probability there is percolation in $\eta_{\mathbb{Z}^{d} \backslash R_{J}}$ (and we are done) or else the convergence of the series analogous to that in (2.5) implies that, with $\nu_{J, p^{-}}$ probability 1 , there are only finitely many separating dual hypersurfaces and percolation occurs.

Finally, we discuss the case in which $J$ is unbounded. The problem is then to show that when $p$ is small enough percolation does not occur for $P$ almost all $J$, in spite of the high density of occupied bonds. In fact, this is the case even if $J$ can take the value $\pm \infty$ with a positive but not too high probability; to include this case into the discussion let us define bond occupation variables for the bonds $b$ such that $J_{b}= \pm \infty$ only if $P\left(\left|J_{b}\right|=\infty\right)<p_{c}(d)$. In this case the definition is the natural one obtained when taking all $\infty$ values to be equivalent and is given as follows: the bonds $b$ such that $\left|J_{b}\right|=\infty$ form finite clusters, and in each of these clusters $C$ there is some subset $S$ which is unfrustrated; let us occupy one such subset at random among all such subsets of maximal cardinality. 
THEOREM 4.4. Consider a conditionally unfrustrated Bernoulli model or an $F K$ random cluster model on $\mathbb{Z}^{d}, d \geq 2$ (or on the triangular lattice $T$ ). If there exists $M>0$ such that $\mathscr{P}\left(\left|J_{b}\right|>M\right)=\varepsilon<p_{c}(d)$ [or $p_{c}(T)$, respectively] then if $p>0$ is such that

$$
\varepsilon+\left(1-(1-p)^{M}\right)(1-\varepsilon)<p_{c}(d) \quad\left[o r p_{c}(T)\right],
$$

percolation does not occur for $\mathscr{P}$-almost all $\mathrm{J}$.

Proof. Without regard to the values taken by a configuration $\eta$ on the bonds $b$ in which $\left|J_{b}\right|>M$, for each $b$ such that $\left|J_{b}\right| \leq M$, it follows from the definition in (4.1) that

$$
\sup _{\eta_{\backslash b}} \nu_{J, p}\left(\eta_{b}=1 \mid \eta_{\backslash b}\right) \leq\left(1-(1-p)^{M}\right) .
$$

With $\mathscr{P}$-probability 1 the density of bonds $b$ such that $\left|J_{b}\right|>M$ does not exceed $\varepsilon$ and these are chosen independently from one another. By assuming now that these are all occupied, we get that for all bonds $b$,

$$
\sup _{\eta_{\backslash b}} \nu_{J, p}\left(\eta_{b}=1 \mid \eta_{\backslash b}\right) \leq \varepsilon+(1-\varepsilon)\left(1-(1-p)^{M}\right) ;
$$

if (4.9) holds $\nu_{J, p}$ is dominated by a Bernoulli measure with parameter less than $p_{c}(d)$ [or $p_{c}(T)$ ]. This implies that percolation does not occur under $\nu_{J, p}$ for all such $J$ 's, that is, with $\mathscr{P}$-probability 1.

Acknowledgment. The authors thank an anonymous referee for general comments and specific suggestions.

\section{REFERENCES}

[1] Aizenman, M., Chayes, J. T., Chayes, L., Frölich, J. and Russo, L. (1983). On a sharp transition from area law to perimeter law in a system of random surfaces. Comm. Math. Phys. 92 19-69.

[2] Aizenman, M. and WeHR, J. (1990). Rounding effect of quenched randomness on first-order phase transitions. Comm. Math. Phys. 130 489-528.

[3] Alexander, K. (1995). Simultaneous uniqueness of infinite cluster in stationary random labeled graphs. Comm. Math. Phys. 168 39-55.

[4] BaXter, R. J. (1982). Exactly Solved Models in Statistical Mechanics. Academic Press, London.

[5] Campanino, M. (1998). Strict inequality for critical percolation values in frustrated random/cluster models. Markov Processes and Related Fields 4 395-410.

[6] Chayes, J. T., Chayes, L. and Frölich, J. (1985). The low-temperature behaviour of disordered magnets. Comm. Math. Phys. 100 399-437.

[7] De SANTis, E. (1998). Strict inequalities in phase transition between ferromagnetic and frustrated systems. Ph.D. thesis, Rome.

[8] Edwards, S. and Anderson, P. W. (1975). Theory of spin glasses. J. Phys. F. 5 965-974.

[9] Fontes, L. and Newman, C. M. (1993). First passage percolation for random colorings of $\mathbb{Z}^{d}$. Ann. Appl. Probab. 3 746-762.

[10] Fortuin, C. M. and KASTEleyn, P. W. (1972). On the random-cluster model I. Introduction and relation to other models. Physica 57 536-564.

[11] Fortuin, C. M., Kasteleyn, P. W. and Ginibre, J. (1971). Correlation inequalities on some partially ordered set. Comm. Math. Phys. 22 89-103. 
[12] Gandolfi, A. (1999). A remark on gauge symmetries in Ising spin glasses. Probab. Theory Related Fields 144 419-430.

[13] Gandolfi, A., Keane, M. S. and Newman, C. M. (1992). Uniqueness of the infinite component in a random graph with applications to percolation and spin glasses. Probab. Theory Related Fields 92 511-527.

[14] Grimmett, G. (1990). Percolation. Springer, New York.

[15] Grimmett, G. and Marstrand, J. M. (1990). The supercritical phase of percolation is well behaved. Proc. Roy. Soc. London Ser. A $430439-457$.

[16] Haggström, O., Peres, Y. and Steif, J. E. (1997). Dynamical percolation. Ann Inst. H. Poincaré Probab. Statist. 33 491-495.

[17] HARRIS, T. E. (1960). A lower bound for the critical probability in a certain percolation process. Math. Proc. Cambridge Philos. Soc. 56 13-20.

[18] Kesten, H. (1980). The critical probability of bond percolation on the square lattice equals 1/2. Comm. Math. Phys. 74 41-59.

[19] Kesten, H. (1982). Percolation Theory for Mathematicians. Birkhäuser, Boston.

[20] Lebowitz J. L. and Mazel, A. E. (1998). Improved Peierls argument for high dimensional Ising models. J. Statist. Phys. 90 1051-1059.

[21] LE GALL, J.-F. (1992). Some properties of planar Brownian motion. Ecóle d'éte de probabilités de Saint-Flour XX. Lecture Notes in Math. 1527 111-235. Springer, New York.

[22] Liggett, T. M., Schonmann, R. H. and Stacey, A. M. (1997). Domination by product measures. Ann. Probab. 25 71-95.

[23] Newman, C. M. (1994). Disordered Ising systems and random cluster representation. In Probability and Phase Transitions (G. Grimmett, ed.) 247-260. Kluwer, Dordrecht.

[24] Newman, C. M. (1997). Topics in Disordered Systems. Birkhäuser, Berlin.

[25] Newman, C. M. and Stein, D. L. (1996). Ground-states structure in a highly disordered spin-glass model. J. Statist. Phys. 82 1113-1132.

[26] Newman, C. M. and Stein, D. L. (1996). Non-mean-field behaviour of realistic spin glasses. Phys. Rev. Lett. 76 515-518.

[27] ShePp, L. A. (1972). Covering the circle with random arcs. Israel J. Math. 11 328-345.

DiPARTIMENTO DI MATEMATICA

UNIVERSITÀ DI ROMA LA SAPIENZA

PiAzZAle A. Moro, 2

00185 RoMA

ITALY
DiPARTIMENTO DI MATEMATICA UNIVERSITÀ DI ROMA TOR VERGATA VIALE DELLA RICERCA SCIENTIFICA 00133 ROMA

ITALY 